Accepted by ApJ December 14, 2010

\title{
Formation of collapsing cores in subcritical magnetic clouds: three-dimensional MHD simulations with ambipolar diffusion
}

\author{
Takahiro Kudoh ${ }^{1}$ and Shantanu Basu ${ }^{2}$
}

\begin{abstract}
We employ the three-dimensional magnetohydrodynamic simulation including ambipolar diffusion to study the gravitationally-driven fragmentation of subcritical molecular clouds, in which the gravitational fragmentation is stabilized as long as magnetic flux-freezing applies. The simulations show that the cores in an initially subcritical cloud generally develop gradually over an ambipolar diffusion time, which is about a few $\times 10^{7}$ years in a typical molecular cloud. On the other hand, the formation of collapsing cores in subcritical clouds is accelerated by supersonic nonlinear flows. Our parameter study demonstrates that core formation occurs faster as the strength of the initial flow speed in the cloud increases. We found that the core formation time is roughly proportional to the inverse of the square root of the enhanced density created by the supersonic nonlinear flows. The density dependence is similar to that derived in quasistatically contracting magnetically supported clouds, although the core formation conditions are created by the nonlinear flows in our simulations. We have also found that the accelerated formation time is not strongly dependent on the initial strength of the magnetic field if the cloud is highly subcritical. Our simulation shows that the core formation time in our model subcritical clouds is several $\times 10^{6}$ years, due to the presence of large-scale supersonic flows $(\sim 3$ times sound speed). Once a collapsing core forms, the density, velocity, and magnetic field structure of the core does not strongly depend on the initial strength of the velocity fluctuation. The infall velocities of the cores are subsonic and the magnetic field lines show weak hourglass shapes.
\end{abstract}

Subject headings: ISM: clouds, ISM: magnetic fields, stars: formation

\footnotetext{
${ }^{1}$ Division of Theoretical Astronomy, National Astronomical Observatory, 2-21-1 Osawa, Mitaka, Tokyo 181-8588, Japan

${ }^{2}$ Department of Physics and Astronomy, University of Western Ontario, London, Ontario N6A 3K7, Canada
} 


\section{Introduction}

Magnetic fields in molecular clouds play an important role in the early stage of star formation. Particularly, magnetic fields in molecular clouds can regulate the cloud collapse and fragmentation process. The important parameter for these processes is the mass-to-flux ratio $M / \Phi$, where $M$ is the mass and $\Phi$ is the magnetic flux of the cloud. The mass-to-flux ratio represents the relative strength of gravity and the magnetic field. There exists a critical massto-flux ratio $(M / \Phi)_{\text {crit }}$ (Mestel \& Spitzer 1956; Strittmatter 1966; Mouschovias \& Spitzer 1976; Tomisaka, et al. 1988). If $M / \Phi>(M / \Phi)_{\text {crit }}$, the cloud is supercritical and is prone to collapse. On the other hand, if $M / \Phi<(M / \Phi)_{\text {crit }}$, a cloud is subcritical and cannot collapse as long as magnetic flux-freezing applies. A similar condition $M / \Phi<(M / \Phi)_{\text {crit }}=1 /\left(2 \pi G^{1 / 2}\right)$ is required for stability against fragmentation of an infinite uniform layer that is flattened along the direction of a background magnetic field (Nakano \& Nakamura 1978), where $G$ is the gravitational constant.

Mestel \& Spitzer (1956) pointed out that even if clouds are magnetically supported, ambipolar diffusion will cause the support to be lost and collapse will begin. More generally, a subcritical cloud undergoes a gravitationally-driven fragmentation instability that occurs on the ambipolar diffusion timescale rather than the dynamical timescale (Langer 1978; Zweibel 1998; Ciolek \& Basu 2006). The lengthscale of the instability is fundamentally the Jeans-scale in the limit of highly supercritical clouds, but can be much larger when the mass-to-flux ratio is close to the critical value (Ciolek \& Basu 2006). The idea that the star formation is regulated by ambipolar diffusion and the magnetic field has been considered for many years (e.g., Shu et al. 1987, 1999; Mouschovias \& Ciolek 1999).

Magnetic field strength measurements through the Zeeman effect reveal that the massto-flux ratios of cloud cores are close to the critical value (Crutcher 2004). These observations are consistent with core formation driven by ambipolar diffusion in subcritical clouds. However, in order to assess whether the fragmentation is occurring in a subcritical or supercritical molecular cloud, magnetic field measurements of the envelope or diffuse region are important. Crutcher et al. (2009) recently attempted to do this using the Zeeman effect, and argued that the mass-to-flux ratio of the envelope (in four measured positions with beam diameters in the range of $\sim 0.5-1 \mathrm{pc}$ in the vicinity of four different cloud cores) is typically greater than that of the core. This contradicts the model of core formation in subcritical clouds. However, Mouschovias \& Tassis (2009) contested their statistical analysis, and argued that dropping the restrictive assumption that the magnetic field is constant across four measured envelope regions of differing morphology and density would result in the opposite conclusion, i.e., that the core mass-to-flux ratio is likely greater than that of the envelope. Future observational tests of this kind, but benefiting from increased amounts of source 
integration time and spatial resolution, can settle the current difficulties of interpretation (Crutcher et al. 2010). The complementary method of measurements of polarized emission from dust grains, which reveal the magnetic field morphology in the cloud, generally show that the magnetic field in cloud cores is well ordered, and application of the ChandrasekharFermi method yields mass-to-flux ratios that are also near the critical value (e.g., Schleuning 1998; Girart et al. 2006). Li et al. (2009) recently compared the magnetic field directions on core scales $(<1 \mathrm{pc})$ with those on large scales $(>200 \mathrm{pc})$ for several molecular clouds, and found a significant correlations. Alves et al. (2008) distinctly shows that the magnetic field is locally perpendicular to the large filamentary structure of the Pipe Nebula. These recent observations of polarized emission indicate that the magnetic field provides a dominant force, and that core formation may have been driven by ambipolar diffusion in subcritical clouds.

Most nonlinear calculations of ambipolar-diffusion-driven evolution in subcritical clouds have focused on a single axisymmetric core, but some recent models focus on a fragmentation process that results in multiple cores. Nonlinear calculations of ambipolar-diffusion-driven fragmentation in subcritical clouds were first performed by Indebetouw \& Zweibel (2000), who carried out a two-dimensional simulation of an infinitesimally thin sheet threaded by an initially perpendicular magnetic field. Basu \& Ciolek (2004) and Basu et al. (2009a) carried out two-dimensional simulations of a magnetized sheet in the thin-disk approximation, which incorporates a finite disk half-thickness consistent with hydrostatic equilibrium and thereby includes the effect of magnetic pressure. They found that the fragment spacing in the nonlinear phase agrees with the prediction of linear theory (Ciolek \& Basu 2006), and that the the subcritical (or critical) model had subsonic infall, while the supercritical model had supersonic infall speed. The first three-dimensional simulation of the gravitational fragmentation with magnetic fields and ambipolar diffusion was performed by Kudoh et al. (2007), which verified some of main results of the thin-disk models: the dichotomy between subsonic infall speeds in subcritical clouds and somewhat supersonic speeds in supercritical clouds, for example.

Besides the magnetic field, the supersonic turbulence in molecular clouds is also an important component in the early stage of star formation (MacLow \& Klessen 2004; McKee \& Ostriker 2007). The inclusion of supersonic turbulent initial conditions to a fragmentation model in subcritical clouds was studied by Li \& Nakamura (2004) and Nakamura \& Li (2005), adopting the thin-disk approximation. They found that a mildly subcritical cloud can undergo locally rapid ambipolar diffusion and form multiple fragments because of the initial supersonic motion in which the large scale wave mode dominates the power spectrum. The core formation occurs on the order of turbulence crossing time over the simulation box, which is comparable to the dynamical time scale. Basu et al. (2009b) carried out a parameter study of the fragmentation regulated by gravity, magnetic fields, ambipolar diffusion, and nonlinear 
turbulent flows. They confirmed the onset of runaway collapse in subcritical cloud is significantly accelerated by the initial nonlinear flows. Kudoh \& Basu (2008) also verified that the mode of turbulence-accelerated magnetically-regulated star formation occurs in a fully three-dimensional simulation. Nakamura \& Li (2008) also carried out three-dimensional MHD simulations of subcritical clouds including star star formation and bipolar outflows, and applied their model to the Taurus molecular cloud. Since three-dimensional simulations of subcritical clouds are resource-limited, large parameter studies have not yet been performed.

In this paper, we study the fragmentation process in subcritical clouds, including ambipolar diffusion, by fully three-dimensional simulations. Our study follows the previous

ones of Kudoh et al. (2007) and Kudoh \& Basu (2008). We focus on the early stage of core formation and evolution, and do not follow evolution past the runaway collapse of a core. We carry out a parameter study by running a large number of models, and with higher spatial resolution than in our previous studies. We are especially interested in the effect of the large-amplitude nonlinear initial perturbations on the time evolution of the cloud fragmentation, and discuss the mechanism of turbulence-accelerated star formation in subcritical clouds.

Our paper is organized in the following manner. The numerical model is described in Section 2, results are given in Section 3, and a discussion of the results is given in Section 4. We summarize our results in Section 5.

\section{Numerical model}

The numerical model used in this paper is almost the same as that used by Kudoh et al. (2007) and Kudoh \& Basu (2008). We solve the three-dimensional magnetohydrodynamic (MHD) equations including self-gravity and ambipolar diffusion. As an initial condition, we assume hydrostatic equilibrium of a self-gravitating cloud along the direction of an initially uniform magnetic field. We also assume an initially uniform structure perpendicular to the magnetic field lines. In this equilibrium sheet-like gas, we input a random velocity fluctuation at each grid point.

\subsection{Basic Equations}

We solve the three-dimensional MHD equations including self-gravity and ambipolar diffusion, assuming that neutrals are much more numerous than ions: 


$$
\begin{gathered}
\frac{\partial \rho}{\partial t}+\boldsymbol{v} \cdot \nabla \rho=-\rho \nabla \cdot \boldsymbol{v}, \\
\frac{\partial \boldsymbol{v}}{\partial t}+(\boldsymbol{v} \cdot \nabla) \boldsymbol{v}=-\frac{1}{\rho} \nabla p+\frac{1}{c \rho} \boldsymbol{j} \times \boldsymbol{B}-\nabla \psi, \\
\frac{\partial \boldsymbol{B}}{\partial t}=\nabla \times(\boldsymbol{v} \times \boldsymbol{B})+\nabla \times\left[\frac{\tau_{n i}}{c \rho}(\boldsymbol{j} \times \boldsymbol{B}) \times \boldsymbol{B}\right], \\
\boldsymbol{j}=\frac{c}{4 \pi} \nabla \times \boldsymbol{B}, \\
\nabla^{2} \psi=4 \pi G \rho, \\
p=c_{s}^{2} \rho,
\end{gathered}
$$

where $\rho$ is the density of neutral gas, $p$ is the pressure, $\boldsymbol{v}$ is the velocity, $\boldsymbol{B}$ is the magnetic field, $\boldsymbol{j}$ is the electric current density, $\psi$ is the self-gravitating potential, and $c_{s}$ is the sound speed. Here, we consider the molecular clouds whose number density is about $10^{3}-10^{6}$ $\mathrm{cm}^{-3}$. In this case, the cooling time is generally much smaller than the dynamical time. Therefore, instead of solving a detailed energy equation, we assume isothermality for each Lagrangian fluid particle (Kudoh \& Basu 2003, 2006):

$$
\frac{d c_{s}}{d t}=\frac{\partial c_{s}}{\partial t}+\boldsymbol{v} \cdot \nabla c_{s}=0 .
$$

This means that each parcel of molecular cloud gas as well as surrounding warm gas (see Sec. 2.2) retains its initial temperature. For the neutral-ion collision time in equation (3) and associated quantities, we follow Basu \& Mouschovias (1994), so that

$$
\tau_{\mathrm{ni}}=1.4 \frac{m_{i}+m_{n}}{\rho_{i}\langle\sigma w\rangle_{i n}},
$$

where $\rho_{i}$ is the density of ions and $\langle\sigma w\rangle_{\text {in }}$ is the average collisional rate between ions of mass $m_{i}$ and neutrals of mass $m_{n}$. Here, we use typical values of $\mathrm{HCO}^{+}-\mathrm{H}_{2}$ collisions, for which $\langle\sigma w\rangle_{\text {in }}=1.69 \times 10^{-9} \mathrm{~cm}^{-3} \mathrm{~s}^{-1}, m_{\mathrm{i}} / m_{\mathrm{n}}=14.4$, and $m_{\mathrm{n}}=2 \times 1.66 \times 10^{-24} \mathrm{~g}$. We also assume that the ion density $\rho_{i}$ is determined by the approximate relation (Elmegreen 1979; Nakano 1979)

$$
\rho_{\mathrm{i}}=m_{\mathrm{i}} K\left(\frac{\rho / m_{\mathrm{n}}}{10^{5} \mathrm{~cm}^{-3}}\right)^{k},
$$


where we assume $k=0.5$ throughout this paper, and take $K=3 \times 10^{-3} \mathrm{~cm}^{-3}$ as a typical value. By using equation (9), equation (8) can be written as

$$
\tau_{\mathrm{ni}}=\gamma \rho^{-1 / 2}
$$

where $\gamma \simeq 170.2 \mathrm{~g}^{1 / 2} \mathrm{~cm}^{-3 / 2} \mathrm{~s}$ using the above typical values. Then, equation (3) becomes

$$
\frac{\partial \boldsymbol{B}}{\partial t}=\nabla \times(\boldsymbol{v} \times \boldsymbol{B})+\nabla \times\left[\frac{\gamma}{c \rho^{3 / 2}}(\boldsymbol{j} \times \boldsymbol{B}) \times \boldsymbol{B}\right] \text {. }
$$

\subsection{Initial Conditions}

As an initial condition, we assume hydrostatic equilibrium of a self-gravitating onedimensional cloud along the $z$-direction (Kudoh \& Basu 2003, 2006). The hydrostatic equilibrium is calculated from the equations

$$
\begin{gathered}
\frac{d p}{d z}=\rho g_{z}, \\
\frac{d g_{z}}{d z}=-4 \pi G \rho, \\
p=c_{s}^{2} \rho,
\end{gathered}
$$

subject to the boundary conditions

$$
g_{z}(z=0)=0, \quad \rho(z=0)=\rho_{0}, \quad p(z=0)=\rho_{0} c_{s 0}^{2}
$$

where $\rho_{0}$ and $c_{s 0}$ are the initial density and sound speed at $z=0$. If the initial sound speed (temperature) is uniform throughout the region, we have the following analytic solution $\rho_{S}$ found by Spitzer (1942):

$$
\rho_{S}(z)=\rho_{0} \operatorname{sech}^{2}\left(z / H_{0}\right)
$$

where

$$
H_{0}=\frac{c_{s 0}}{\sqrt{2 \pi G \rho_{0}}}
$$

is the scale height. However, an isothermal molecular cloud is usually surrounded by warm

material, such as neutral hydrogen gas. Observations also show that the transition between 
molecular gas and surrounding gas is quite sharp Blitz 1991). In order to simulate the situation, we assume the initial sound speed distribution to be

$$
c_{s}^{2}(z)=c_{s 0}^{2}+\frac{1}{2}\left(c_{s c}^{2}-c_{s 0}^{2}\right)\left[1+\tanh \left(\frac{|z|-z_{c}}{z_{d}}\right)\right],
$$

where we take $c_{s c}^{2}=10 c_{s 0}^{2}, z_{c}=2 H_{0}$, and $z_{d}=0.1 H_{0}$ throughout the paper. This equation gives a numerical model of the temperature transition of $c_{s c}^{2} / c_{s 0}^{2}$ at $z_{c}$ with the transition length of $z_{d}$. By using this sound speed distribution, we can solve equations (12)-(14) numerically. The initial density distribution of the numerical solution shows that it is almost the same as Spitzer's solution for $0 \leq z \leq z_{c}$.

We also assume that the initial magnetic field is uniform along the $z$-direction:

$$
B_{z}=B_{0}, \quad B_{x}=B_{y}=0
$$

where $B_{0}$ is constant.

In this equilibrium sheet-like gas, we input a random velocity fluctuation at each grid point:

$$
v_{x}=v_{a} R_{m}(x, y), \quad v_{y}=v_{a} R_{m}(x, y), \quad v_{z}=0.0,
$$

where $R_{m}$ is a random number with spectrum $v_{k}^{2} \propto k^{n}$ in Fourier space, where $k=\left(k_{x}^{2}+k_{y}^{2}\right)^{1 / 2}$, and the root mean square value is about 1 . The $R_{m}$ 's for each of $v_{x}$ and $v_{y}$ are independent realizations. In this paper, we apply the two distinct spectra of $v_{k}^{2} \propto k^{4}$ and $v_{k}^{2} \propto k^{0}$. The parameter $v_{a}$ shows the strength of the velocity fluctuation. Models with the same spectrum use the same pair of realizations of $R_{m}$ for generating the initial perturbations.

\subsection{Numerical Parameters}

The constant $G$ along with the two parameters $c_{s 0}$ and $\rho_{0}$ that are associated with the initial state allow one to choose a set of three fundamental units for this problem. We choose these to be $c_{s 0}, H_{0}$, and $\rho_{0}$ for velocity, length, and density, respectively. These yield a time unit $t_{0} \equiv H_{0} / c_{s 0}$. The initial magnetic field strength introduces one dimensionless free parameter,

$$
\beta_{0} \equiv \frac{8 \pi p_{0}}{B_{0}^{2}}=\frac{8 \pi \rho_{0} c_{s 0}^{2}}{B_{0}^{2}},
$$

the initial ratio of gas to magnetic pressure at $z=0$.

In the sheet-like equilibrium cloud with a vertical magnetic field, $\beta_{0}$ is related to the mass-to-flux ratio for Spitzer's self-gravitating cloud. The mass-to-flux ratio normalized to 
the critical value is

$$
\mu_{S} \equiv 2 \pi G^{1 / 2} \frac{\Sigma_{S}}{B_{0}}
$$

where

$$
\Sigma_{S}=\int_{-\infty}^{\infty} \rho_{S} d z=2 \rho_{0} H_{0}
$$

is the column density of Spitzer's self-gravitating cloud. Therefore,

$$
\beta_{0}=\mu_{S}^{2}
$$

Although the initial cloud we used is not exactly the same as the Spitzer cloud, $\beta_{0}$ is a good indicator to whether or not the magnetic field can prevent gravitational instability in the flux-freezing case (Nakano \& Nakamura 1978).

We also define a dimensionless parameter

$$
\alpha \equiv \gamma \sqrt{2 \pi G},
$$

which shows the strength of the ambipolar diffusion. We note that $\alpha$ can also be equated to $\tau_{\mathrm{ni}, 0} / t_{0}$, where $\tau_{\mathrm{ni}, 0}$ is the initial value of the neutral-ion collision time calculated from equation (10). By using the typical values in Section 2.1, we get $\alpha \simeq 0.11$, which is taken as a fiducial parameter in this paper. We also vary $\alpha$ as a free parameter in Section 3.3.

Dimensional values of all quantities can be found through a choice of $\rho_{0}$ and $c_{s 0}$. For example, for $c_{s 0}=0.2 \mathrm{~km} \mathrm{~s}^{-1}$ and $n_{0}=\rho_{0} / m_{n}=10^{4} \mathrm{~cm}^{-3}$, we get $H_{0} \simeq 0.05 \mathrm{pc}, t_{0} \simeq$ $2.5 \times 10^{5} \mathrm{yr}$, and $B_{0} \simeq 20 \mu \mathrm{G}$ if $\beta_{0}=1$.

\subsection{Numerical Technique}

In order to solve the equations numerically, we use the CIP method (Yabe \& Aoki 1991; Yabe et al. 2001) for equations (11), (2) and (7), and the method of characteristicsconstrained transport (MOCCT; Stone \& Norman 1992) for equation (3), including an explicit integration of the ambipolar diffusion term. The combination of the CIP and MOCCT methods is summarized in Kudoh et al. (1999) and Ogata et al. (2004). It includes the CCUP method (Yabe \& Wang 1991) for the calculation of gas pressure, in order to get more numerically stable results. The numerical code used for this paper is based on that of Ogata et al. (2004).

In this paper, the ambipolar diffusion term is only included when the density is greater than a certain value, $\rho_{c r}$. We let $\rho_{c r}=0.3 \rho_{0}$ both for numerical convenience and due to 
the physical idea that the low density region is affected by external ultraviolet radiation so that the ionization fraction becomes large, i.e., $\tau_{\text {ni }}$ becomes small (Ciolek \& Mouschovias 1995). Under this assumption, the upper atmosphere of the sheet-like cloud is not affected by ambipolar diffusion. This simple assumption helps to avoid very small time-steps due to the low density region in order to maintain stability of the explicit numerical scheme.

We use a mirror-symmetric boundary condition at $z=0$ and periodic boundaries in the $x$ - and $y$-directions. At the upper boundary, at $z=z_{\text {out }}=4 H_{0}$, we also use a mirrorsymmetric boundary except when we solve for the gravitational potential. This symmetric condition is just for numerical convenience. When the same boundary conditions were used by Kudoh et al. (2007) and Kudoh \& Basu (2008) for three-dimensional simulations, the re-

sults were consistent with two-dimensional thin-disk simulations (e.g., Basu \& Ciolek 2004; Basu et al. 2009b). Hence, we believe that the boundary conditions do not affect the result significantly. The Poisson equation (5) is solved by the Green's function method to compute the gravitational kernels in the $z$-direction, along with a Fourier transform method in the $x$ and $y$-directions (Mivama et al. 1987b). This method of solving the Poisson equation allows us to find the gravitational potential of a vertically isolated cloud within $|z|<z_{\text {out }}$.

The computational region is $|x|,|y| \leq 8 \pi H_{0}$ and $0 \leq z \leq 4 H_{0}$. The number of grid points for each direction is $\left(N_{x}, N_{y}, N_{z}\right)=(256,256,40)$. Since the most unstable wavelength for no magnetic field is about $4 \pi H_{0}$ (Miyama et al. 1987a), we have 64 grid points within this wavelength. We have also 10 grid points within the scale height of the initial cloud in the $z$-direction. The computational time of the fiducial model is about 70 hours of CPU time using four processors of the SX-9 in the National Astronomical Observatory of Japan. The maximum computational time, which occurs for the case of the highly subcritical cloud with small initial velocity fluctuations, is about 770 hours of CPU time. In the cases of the supercritical model, the computational times are about 30 minutes of CPU time.

\section{Results}

Table 1 summarizes the models and parameters for simulations presented in this paper. In the table, we have listed the values of the free parameters $\beta_{0}, \alpha$, the form of the turbulent power spectrum, and $v_{a}$. We also have listed the core formation time $t_{\text {core }}$, which is defined at the time when the density of the core reaches $100 \rho_{0}$. Although the definition of $t_{\text {core }}$ is determined from a practical constraint of the numerical simulation, in practice the center of the core is always supercritical at this time and the time evolution of the density shows the features of runaway collapse. In the models V0 to V5, we change the amplitude of the initial velocity fluctuation $v_{a}$, with the form of the turbulent power spectrum fixed at $v_{k}^{2} \propto k^{-4}$. In 
the models $\mathrm{K} 0$ to $\mathrm{K} 4$, we change the amplitude of the initial velocity fluctuation, but with the form of the turbulent power spectrum fixed at $v_{k}^{2} \propto k^{0}$. In the models $\mathrm{A} 0$ to $\mathrm{A} 4$, we change the dimensionless ambipolar diffusion coefficient $\alpha$ for models with $v_{a}=3.0 c_{s 0}$ or $v_{a}=0.1 c_{s 0}$. In the models $\mathrm{B} 0$ to $\mathrm{B} 9$, we change $\beta_{0}$, the initial plasma beta at $z=0$, which corresponds to the square of the initial mass-to-flux ratio, for models with $v_{a}=3.0 c_{s 0}$ or $v_{a}=0.1 c_{s 0}$. We are mostly interested in core formation in subcritical clouds, but also carry out some supercritical cases for comparison.

\subsection{General properties of a fiducial model}

We show the result of model V4 as a fiducial model, in which the initial turbulent velocity amplitude $\left(v_{a}\right)$ is 3 times sound speed $\left(c_{s 0}\right)$, its spectrum is $v_{k}^{2} \propto k^{-4}$, the initial normalized mass-to-flux ratio is about 0.5 (i.e., $\beta_{0}=0.25$ ), and the dimensionless ambipolar diffusion coefficient has a typical value $(\alpha=0.11)$. We suppose that these values are approximately typical in molecular clouds.

Figure 1 shows the time snapshot of the logarithmic density colour map overlaid with the velocity vector field for model V4. The snapshot is at the end of our simulation, when the maximum density is greater than $100 \rho_{0}$. The upper panel shows the cross section at $z=0$, and the lower panel shows it at $y=20.7 H_{0}$. The maximum density is located at $(x, y, z)=\left(-13.4 H_{0}, 20.7 H_{0}, 0\right)$. A collapsing core is located in the vicinity of the maximum density. Figure 2 shows the time snapshot of the logarithmic plasma $\beta$, the ratio of gas pressure to magnetic pressure, at the end of the simulation. It shows that $\beta$ is greater than 1 around the core. It means that once the collapsing core is formed, the mass-to-flux ratio of the core is expected to be greater than $1(\beta>1)$, i.e., supercritical. The other collapsing cores are also formed in the region where plasma $\beta$ is greater than 1 (the left-down region of the upper panel in Fig. 2). The left panel of Figure 3 shows the density, $x$-velocity, and plasma $\beta$ along an $x$-axis cut at $y=20.7 H_{0}$ and $z=0$. The right panel shows the density, $z$-velocity, and plasma $\beta$ along a $z$-axis cut at $x=-13.4 H_{0}$ and $y=20.7 H_{0}$. In each panel, the velocity shows infall motion into the center of the core, though the $x$-velocity outside of the core (low density region) is affected by turbulent motions originating from the initial perturbation. The relative infall speed is subsonic and is about half of the sound speed at that time. The subsonic infall feature is consistent with previous studies of core formation in subcritical clouds (e.g., Ciolek \& Basu 2000; Basu \& Ciolek 2004).

Figure 4 shows the time evolution of the maximum density at $z=0$ and $\beta$ at the location of the maximum density. The density goes up to $\sim 10$ times greater than the initial density during the first compression of the cloud. Then, it rebounds and shows oscillations. 
Eventually, the dense region goes into runaway collapse around $t=18 t_{0}$, which corresponds to about $5 \times 10^{6}$ years. In the first compression, $\beta$ also goes up to $\sim 0.6$. It gradually goes up and becomes greater than 1 when the runaway collapse occurs. As we discussed in the previous section, $\beta$ roughly equals the square of the normalized mass-to-flux ratio in the gravitational equilibrium state of gas with vertical magnetic field. The runaway collaping core is expected to be supercritical $(\beta>1)$. Figure 4, Figure 2, and Figure 3 demonstrate that

$\beta$ is a good indicator of the mass-to-flux ratio, though the gas is not exactly in gravitational equilibrium at the last stage of our simulations.

Figure 5 shows a close-up view of the core in the vicinity of $(x, y, z)=\left(-13.4 H_{0}, 20.7 H_{0}, 0\right)$. The iso-surface contour shows the logarithmic density and the lines represent the magnetic field. The core is located in a filamentary structure that was induced by the initial velocity fluctuation. The magnetic field lines show an hourglass shaped structure because of the infall motion into the center of the core.

\subsection{Effect of initial velocity fluctuations}

Figure 6 shows a time snapshot of the logarithmic density overlaid with velocity vectors for model V0. The model V0 coresponds to the model for initial velocity amplitude $\left(v_{a}\right)$ is 0.01 times sound speed $\left(c_{s 0}\right)$, but other parameters are the same as the fiducial model V4. The snapshot is at the end of our simulation, when the maximum density is greater than $100 \rho_{0}$. When the initial perturbed velocity is much smaller than the sound speed, the core formation time $\left(t_{\text {core }} \sim 136 t_{0}\right.$, which corresponds to $\sim 3 \times 10^{7}$ years $)$ is much longer than that $\left(t_{\text {core }} \sim 18.7 t_{0}\right)$ of model V4. The maximum density is located at $(x, y, z)=$ $\left(7.6 H_{0},-11.9 H_{0}, 0\right)$. A collapsing core is formed in the vicinity of the maximum density. Figure 7 shows the time snapshot of the logarithmic plasma $\beta$ at the end of the simulation for model V0. As we expected, the plasma $\beta$ value is greater than 1 around the core, which means the core is supercritical. In Figure 6 and Figure 7, core-like structures are gathered together on the side of $x>0$. This reflects the initial perturbation of $k^{-4}$ in which the larger scale contains greater energy in the velocity spectrum. The left panel of Figure 8 shows the density, $x$-velocity, and plasma $\beta$ along an $x$-axis cut at $y=7.6 H_{0}$ and $z=0$. The right panel shows the density, $z$-velocity, and plasma $\beta$ along a $z$-axis cut at $x=-7.6 H_{0}$ and $y=-11.9 H_{0}$. The velocity shows infall motion into the center of the core, though the center of the core shows a systematic speed in the positive $x$-direction of about $0.3 c_{s 0}$. The relative infall speed is also subsonic. Figure 9 shows the close up view of the core in the vicinity of $(x, y, z)=\left(-13.4 H_{0}, 20.7 H_{0}, 0\right)$. The iso-surface contour shows the logarithmic density and the lines represent the magnetic field. The core shows typical oblate-like structure. The 
magnetic field line shows a hourglass shaped structure and the deformation of the field line is similar to that of model V4 in Figure 5.

Figure 10 shows the time snapshot of the logarithmic density overlaid with velocity vectors at the end of the simulation for model K1. The model K1 coresponds to the model for the initial velocity spectrum is $v_{k}^{2} \propto k^{0}$, but other parameters are the same as the fiducial model V4. The flat spectrum is possibly not consistent with observation, but it is used to compare with the result by the typical spectrum of $v_{k}^{2} \propto k^{-4}$. When the initial perturbed velocity has a flat spectrum, the core formation time $\left(t_{\text {core }} \sim 67 t_{0}\right)$ is longer than that $\left(t_{\text {core }} \sim\right.$ $\left.18.7 t_{0}\right)$ of model V4. The maximum density is located at $(x, y, z)=\left(-1.5 H_{0},-9.5 H_{0}, 0\right)$ and a collapsing core is formed in the vicinity of the maximum density. Figure 11 shows the time snapshot of the logarithmic plasma $\beta$ at the end of the simulation for model K1. We note that the $\beta$ is greater than 1 around the core again. The left panel of Figure 12 shows the density, $x$-velocity, and plasma $\beta$ along an $x$-axis cut at $y=-9.5 H_{0}$ and $z=0$. The right panel shows the density, $z$-velocity, and plasma $\beta$ along a $z$-axis cut at $x=-1.5 H_{0}$ and $y=-9.5 H_{0}$. The velocity shows infall motion into the center of the core, and the relative infall speed is also subsonic again. Figure 13 shows a close-up view of the core in the vicinity of $(x, y, z)=\left(-1.5 H_{0},-9.5 H_{0}, 0\right)$. The magnetic field lines also show an hourglass shaped structure. Figure 3, Figure 8 and Figure 12 show the structure of collapsing cores are not so dependent on the initial velocity fluctuations, except that the outer region of the core in model V4 is strongly affected by the large-scale turbulent flow.

The main difference of each model is the core formation time. Figure 14 shows the time evolution of the maximum density for models V4, V0 and $\mathrm{K} 1$. In the case of model V4, which has a $k^{-4}$ spectrum, the maximum density is strongly increased by the compression caused by the large scale supersonic flow. In the case of model K1, the compression occurs in the initial stage, but it is weaker than that of model V4 because the small scale perturbations contain more power than in model V4. In the case of model V0, with small-amplitude initial perturbations, the core formation time is about 7 times longer than that of model V4.

Figure 15 shows the core formation time as a function of the strength of the initial velocity perturbation. As the perturbed velocity amplitude increases, the core formation time decreases for each type of initial spectrum. In the cases of the initial $k^{-4}$ spectrum (filled squares), the core formation times are systematically smaller than those of the initial $k^{0}$ spectrum (open squares). In order to understand the physics of the core formation time, we plot the core formation time as a function of $\rho_{\text {peak }}$ in Figure 16, where $\rho_{\text {peak }}$ is defined as the value of the density peak during the first compression in the time evolution of the maximum density on $z=0$. In the cases of the $k^{-4}$ spectrum (filled squares), $\rho_{\text {peak }}$ is greater than in the cases of the $k^{0}$ spectrum (open squares), even when $v_{a}$ is relatively small. Figure 
16 shows that the core formation time is shorter when the density peak $\rho_{\text {peak }}$ is greater, and indicates that it is nearly proportional to $1 / \sqrt{\rho_{\text {peak }}}$. The density dependence is similar to that derived in quasistatically contracting magnetically supported cores, assuming a force balance of the magnetic force and gravity in the core (e.g., Mouschovias \& Ciolek 1999). In our simulation, the density is increased by the compression caused by large-scale nonlinear flows, and a dense region undergoes a rebound and oscillations, which means the core is not in an exact force balance. Nevertheless, it is interesting that the core formation time is nearly proportional to $1 / \sqrt{\rho_{\text {peak }}}$. We discuss this subject further in Section 4.

\subsection{Dependence on ambipolar diffusion coefficient}

Figure 17 shows the time evolution of the maximum density for models V4, A0, A1 and A2. The model A0, A1, and A2 correspond to the models for $\alpha=0, \alpha=0.05$, and $\alpha=0.2$, respectively, but other parameters of them are the same as those of V4.

As $\alpha$ decreases, the core formation time increases. When $\alpha=0$ (model A0), which means no ambipolar diffusion, a core was not formed during the calculation time, lasting until $t \simeq 268 t_{0}$. Figure 18 shows the core formation time as a function of $\alpha$. When the initial velocity perturbation is small, the core formation time shows nearly linear dependence on $\alpha^{-1}$ (open triangles). When the initial perturbation is nonlinear (filled triangles), the dependence on $\alpha^{-1}$ is a little steeper than a linear dependence. For model A2 $(\alpha=0.2$, $v_{a}=3 c_{s 0}$ ), the core formation occurs very rapidly, without showing an oscillation of the maximum density. When $\alpha$ is large enough, the supercritical region can appear quickly after the first compression, resulting in a significantly reduced core formation time.

In figure 19, we show the time evolution of the total kinetic energies in the computational region for model V4, A0, A1, and A2. The oscillation of the energies comes from the strong compression and rebound of the clouds. The figure shows that the kinetic energies quickly decrease within a crossing time of the initial perturbed velocity across the computational region. The $e$-folding time of the energies depends slightly on $\alpha$. As $\alpha$ increases, the $e$ folding time increases. In the case of $\alpha=0$ (model A0), about 10 percent of the initial kinetic energy remains at the end of the calculation $\left(t \simeq 268 t_{0}\right)$. Basu \& Dapp (2010) found that the significant portion $(\sim 1 / 2)$ of the initial kinetic energy remains in the flux-freezing case using a thin-disk approximation. Our result shows that the kinetic energy is more dissipative than in the thin-disk approximation. This is because of the different boundary condition in the vertical direction. We discuss this subject further in Section 4. 


\subsection{Dependence on initial mass-to-flux ratio}

Figure 20 shows the time evolution of the maximum densities for models V4, B0, B1 and B2, showing the difference between models with different $\beta_{0}$. The model B0, B1, and $\mathrm{B} 2$ correspond to the models for $\beta=0.04, \beta=0.09$, and $\beta=0.36$, respectively, but other parameters of them are the same as those of V4. As we have shown in Section $2, \beta_{0}$ approximately equals the square of the initial normalized mass-to-flux ratio of the cloud. The figure shows that the core formation time is longer when $\beta_{0}$ is smaller. The figure also shows that the initial density enhancement is smaller when $\beta_{0}$ is smaller. Figure 21 shows the core formation time as a function of $\beta_{0}$. When $\beta_{0}$ is greater than 1 , the initial cloud is supercritical. In the case of the supercritical clouds, the core formation occurs in dynamical time scale. When the initial velocity is large, a core forms during the first compression. The core formation time of the supercritical clouds does not strongly depend on the initial massto-flux ratio (or $\beta_{0}$ ). When $\beta_{0}$ is smaller than 1 , the initial cloud is subcritical. The core formation times of a subcritical cloud are generally longer than those of a supercritical cloud. In the case of a highly subcritical cloud however, the core formation time does not strongly depend on the initial mass-to-flux ratio. The transition occurs near the critical cloud, $\beta_{0} \sim 1$. This tendency is consistent with that of the growth time from the linear analysis using the thin-disk approximation (Ciolek \& Basu 2006) and with that of the ambipolar diffusion time in quasistatically contracting magnetically supported cores (Mouschovias \& Ciolek 1999). It is interesting that the same tendency is achieved even when the initial velocity fluctuation is nonlinear (filled circles).

\section{Discussion}

\subsection{Timescale of core formation}

One of the problems for core formation in subcritical clouds is that the timescale of the core formation is slower than that is expected from observations (e.g., Jijina et al. 1999). In order to solve this problem, Zweibel (2002) and Fatuzzo \& Adams (2002) pointed out that the ambipolar diffusion rate can be enhanced in a turbulent medium with a fluctuating magnetic field. In a different way, Li \& Nakamura (2004) and Nakamura \& Li (2005) found that the compression of the cloud by the large-scale turbulent flow shortened the timescale of core formation even in subcritical clouds. Kudoh \& Basu (2008) and this paper followed the latter idea using three-dimensional simulations without a thin-disk approximation. Here, we discuss how the compression shortens the timescale of core formation in subcritical clouds.

Figure 16 indicates that the core formation time is nearly proportional to $1 / \sqrt{\rho_{\text {peak }}}$, 
where $\rho_{\text {peak }}$ is the value of the density peak during the first compression in the time evolution of the maximum density at $z=0$. The ambipolar diffusion time $\left(\tau_{\text {ad }}\right)$ is estimated from equation (11) as $\tau_{\text {ad }} \sim 4 \pi(\sqrt{2 \pi G} / \alpha) \rho^{3 / 2} L^{2} / B^{2}$, where $L$ is the gradient length scale introduced by the turbulent compression. Because the compression by the nonlinear flow is nearly one-dimensional, the magnetic field scales roughly as $B \propto L^{-1}$ within the fluxfreezing approximation. The surface density $\Sigma$ also scales as $\Sigma \propto L^{-1}$. If the compression is rapid enough that vertical hydrostatic equilibrium cannot established, then $\rho \propto \Sigma \propto L^{-1}$, and $\tau_{a d} \propto L^{5 / 2} \propto \rho^{-5 / 2}$ (Elmegreen 2007; Kudoh \& Basu 2008). This means that diffusion can occur quickly if the turbulent compression creates small values of $L$ or large values of $\rho$. This would lead to a rapidly rising value of $\beta$ in Figure 4 at $t / t_{0} \sim 1$. If diffusion is so effective during the first turbulent compression that a dense region becomes supercritical, then it will evolve directly into collapse. Alternatively, the stored magnetic energy of the compressed (and still subcritical) region may lead to reexpansion of the dense region. If reexpansion of the initial compression does occur, then there is enough time for the vertical structure settle back to near-hydrostatic equilibrium. In this case, the density scales roughly like $\rho \propto \Sigma^{2} \propto L^{-2}$, and $\tau_{\text {ad }} \propto L \propto \rho^{-1 / 2}$. The ambipolar diffusion time is also estimated by Mouschovias \& Ciolek (1999) as $\tau_{\text {ad }} \propto \rho^{-1 / 2}$ in quasistatically contracting magnetically supported cores, assuming a radial force balance between gravity and the magnetic Lorentz force. In our simulation, force balance is not exactly achieved, but the time average (of the oscillations) in the cores can be approximately in force balance. Since the first strong compression leads to rapid magnetic flux loss, a higher density region (than the initial value) is rapidly attained and then settles into an approximate force balance. Since the phase of continuing ambipolar diffusion in the oscillating high density region takes longer than the initial compression, the overall time scale of the core formation is approximately proportional to $\rho_{\text {peak }}^{-1 / 2}$. Here, instead of the time-average density, $\rho_{\text {peak }}$ is used as a representitive density of the compressed gas, since it is complicated to determine the time-average density of the moving, oscillating and collapsing high density region. It is interesting that the relation attains even when the $\rho_{\text {peak }}$ is used. We expect that the average density of the compressed overdense region would nearly proportional to the peak density.

The relation means that the density dependence of $\tau_{\text {ad }} \propto \rho^{-1 / 2}$ is the same as that of the free fall time. Figure 16 shows that the actual time of the core formation is about 30 times longer than the free fall time of gas with $\rho_{\text {peak }}$ when $\alpha=0.11$ and $\beta_{0}=0.5$. The vale of the dimensionless coefficient $(\sim 30)$ is expected to be inversely proportinal to $\alpha$ (section 3.3), and not to be strongly dependent on the mass-to-flux ratio except when it is nearly critical (section 3.4). Even when the time-average density is used instead of the peak density, the value of the dimensionless coefficient would not be so different because of the weak dependence of $\rho\left(\propto \rho^{-1 / 2}\right)$. 
In some cases, collapse may occur during the first compression itself or soon thereafter, and the approximate scaling of the core formation time with $\rho_{\text {peak }}^{-1 / 2}$ will not hold. Whether or not this occurs depends not only on the strength of the turbulent compression, but also on the initial mass-to-flux ratio of the cloud (related to $\beta_{0}$ ) and the ambipolar diffusion coefficient $(\alpha)$. In model B2 (a subcritical model that is closest to critical, with normalized initial mass-to-flux ratio $\simeq 0.6$ ), a reexpansion does occur but the collapse starts relatively quickly, in the third oscillation (Fig. 20). In model A2 $(\alpha=0.2)$, which has the poorest neutral-ion coupling of all models, the collapse starts almost in the first compression (Fig. $17)$.

An overall conclusion is that the core formation occurs more rapidly than it would in the initial state due to the elevated value of $\rho$ in the compressed but oscillating region. Since the core formation time is approximately proprtional to $\rho^{-1 / 2}, 10-100$ times desity enhancement is needed to get $3-10$ times shorter core formation time than that of the standard core formation model in subcritical clouds (e.g. Jijina et al. 1999). The observed non-thermal velocity ( $3-10$ times sound speed) is eligible to make such enhancemenr by the compression in the isothermal clouds, if its scale is larger than the Jeans length.

\subsection{Structure of cores}

Even though the core formation time is accelerated by the nonlinear flows, the density, velocity, and magnetic field structure of a core does not strongly depend on the initial strength of the velocity fluctuation (e.g., Fig. 3, Fig. 8, and Fig. 12). The infall velocities of the cores are subsonic and the magnetic field lines show weak hourglass shapes. This result may be consistent with the result that the core formation time is proportional to

$\rho_{\text {peak }}^{-1 / 2}$. Even when core formation is initiated by the initial turbulence, the core properties can be similar to the quasistatically contracting magnetically supported cores discussed by Mouschovias \& Ciolek (1999). The initial turbulence accelerates core formation, but it eventually dissipates in the dense region when the collapsing core is formed. Subsonic infall motions were found by Lee et al. (2001) in an observational survey of starless cores. They found that the typical infall radii are $0.06-0.14 \mathrm{pc}$ and that the infall speed lies in the range of $0.05-0.09 \mathrm{~km} \mathrm{~s}^{-1}$. These values are consistent with our results. The subsonic infall is the common feature of core formation in subcritical clouds, which has been pointed out by 1D axisymmetric (Ciolek \& Basu 2000) and 2D thin-disk (Basu \& Ciolek 2004; Basu et al. 2009a, b) models. Because of the subcritical infall, the hourglass shapes are a little weaker than that of an initially supercritical cloud that shows trans-sonic infall (Kudoh et al. 2007). More quantitative analysis of the field morphological difference and the infall speeds may 
distinguish the mass-to-flux ratio in a molecular cloud.

\subsection{Energy dissipation}

In Figure 19, we showed the time evolution of the total turbulent kinetic energy in isothermal subcritical clouds. It shows that the kinetic energy dissipates efficiently even in the flux-freezing limit $(\alpha=0)$. This is different from the result of Basu \& Dapp (2010). They found that the turbulent kinetic energy persists in an isothermal subcritical cloud in the flux-freezing limit. They showed that the energy dissipation occurs effectively only in the stage of the first nonlinear compression. After that, the turbulent energy persists, and oscillates about an average value. The average value is estimated to be about half of the initial turbulent kinetic energy for the same parameters as those of our fiducial model. On the other hand, our simulation shows that energy dissipation occurs effectively even in the flux-freezing limit; the energy dissipation occurs almost exponentially during several oscillations, although about 12 percent of the initial kinetic energy remains at the final stage. The difference is likely due to the different vertical boundary conditions on the molecular cloud. Basu \& Dapp (2010) use a thin-disk approximation of the cloud, and adopt the current-free condition of the magnetic field outside of the disk, assuming the outside density is negligibly small. We study the problem by three-dimensional MHD and without a thindisk approximation, but assume that the molecular cloud is surrounded by warm gas whose density is 10 times smaller than the molecular cloud. The computational region in our model is $0<z<4 H_{0}$, so does not cover a very large dynamic range of densities. Therefore, future three-dimensional simulations with a larger computational region along the $z$-direction and including low density gas outside of the disk may make for more revealing comparisons with the result of Basu \& Dapp (2010).

\subsection{Additional parameter surveys for numerical accuracy}

In addition to the parameter study in Table 1, we performed simulations of models with the same parameters as models $\mathrm{V} 4$ and V1, but different random realizations of the initial velocity fluctuations. The core formation time for different realizations varies by about $25 \%$ in these samples. The overall evolution and the result of the accelerated core formation by nonlinear flows were not altered by the different initial random realizations. We performed simulations with different spatial resolutions for the model V4. In the case of the highest resolution of $\left(N_{x}, N_{y}, N_{z}\right)=(512,512,40)$, the core formation time is $t_{\text {core }}=14.2 t_{0}$. There is a slight tendency that the core formation time becomes a little shorter for the high 
resolution cases (Kudoh \& Basu 2009). However, we should note that a realization of the random perturbations for the initial velocity fluctuation is also not the same for the different resolutions, because the random perturbations are input on all scales down to the grid scale. At the least, we can say that the result of the accelerated core formation by nonlinear flows is not altered significantly by spatial resolution. We also performed a simulation with a different boundary condition of magnetic fields for the model V4. Instead of the symmetrical boundaty at $z=z_{\text {out }}$, we used the vertical field condition for the magnetic field, i.e., $B_{x}=B_{y}=0$ for $z \geq z_{\text {out }}$. The overall evolution and the result of the accelerated core formation were not also altered by this. The core formation time for the different baounday varies by about $0.2 \%$ in this sample.

\section{Summary}

We have performed fully three-dimensional magnetohydrodynamic simulations of collapsing core formation in molecular clouds with subcritical mass-to-flux ratio, including ambipolar diffusion. Some of our major findings are as follows.

- Core formation in subcritical clouds is generally slow. The core develops gradually over an ambipolar diffusion time. When the initial mass-to-flux ratio is 0.5 times a critical value, the formation time is about $3 \times 10^{7}$ years for an initial midplane number density $10^{4} \mathrm{~cm}^{-3}$.

- The core formation time is shortened by strong velocity fluctuations. When the average strength of the velocity fluctuation is 3 times the sound speed, the formation time is about $5 \times 10^{6}$ years for the same cloud described above.

- The core formation time scales as $t_{\text {core }} \propto 1 / \sqrt{\rho_{\text {peak }}}$, where $\rho_{\text {peak }}$ is the value of the density peak during the first compression in the time evolution of the maximum density. The density dependence is similar to that derived by Mouschovias \& Ciolek (1999).

- In the case of a highly subcritical cloud, the core formation time does not strongly depend on the initial mass-to-flux ratio even when there is strong velocity fluctuation. This tendency is consistent with the result of the linear analysis of thin-disk approximation studied by Ciolek \& Basu (2006).

- Once a core forms, the density, velocity, and magnetic field structure of the core do not strongly depend on the initial strength of the velocity fluctuation. The infall velocities are subsonic and the magnetic field lines show weak hourglass shapes. 
Numerical simulations were done on the SX-9 at the Center for Computational Astro-

physics in National Astronomical Observatory of Japan. S. B. was supported by a Discovery Grant from the Natural Sciences and Engineering Research Council of Canada.

\section{REFERENCES}

Alves, F. O., Franco, G. A. P., \& Girart, J. M. 2008, A\&A, 486, L13

Basu, S., \& Mouschovias, T. Ch. 1994, ApJ, 432, 720

Basu, S., \& Ciolek, G. E. 2004, ApJ, 607, L39

Basu, S., Ciolek, G. E., Dapp, W. B., \& Wurster, J. 2009, NewA, 14, 483

Basu, S., Ciolek, G. E., \& Wurster, J. 2009, NewA, 14, 221

Basu, S., \& Dapp, W. B. 2010, ApJ, 716, 427

Blitz, L. 1991, in NATO ASIC Proc. 342, The Physics of Star Formation and Early Stellar Evolution, ed. C. J. Lada \& N. D. Kylafis (Dordrecht: Kluwer), 3

Ciolek, G. E., \& Mouschovias, T. Ch. 1995, ApJ, 454, 194

Ciolek, G. E., \& Basu, S. 2000, ApJ, 529, 925

Ciolek, G. E., \& Basu, S. 2006, ApJ, 652, 442

Crutcher, R. M. 2004, ApSS, 292, 225

Crutcher, R. M., Hakobian, N., \& Troland, T. H. 2009, ApJ, 692, 844

Crutcher, R. M., Hakobian, N., \& Troland, T. H. 2010, MNRAS, 402, L64

Elmegreen, B. G. 1979, ApJ, 232, 729

Elmegreen, B. G. 2007, ApJ, 668, 1064

Fatuzzo, M., Adams, F. C. 2002, ApJ, 570, 210

Girart, J. M., Rao, R., \& Marrone, D. P. 2006, Science, 313, 812

Indebetouw, R. M., \& Zweibel, E. G. 2000, ApJ, 532, 361

Jijina, J., Myers, P. C., \& Adams, F. C. 1999, ApJS, 125, 161 
Kudoh, T., Matsumoto, R., \& Shibata, K. 1999, Computational Fluid Dynamics Journal, 8, 56

Kudoh, T., \& Basu, S. 2003, ApJ, 595, 842

Kudoh, T., \& Basu, S. 2006, ApJ, 642, 270

Kudoh, T., Basu, S., Ogata, Y., \& Yabe, T. 2007, MNRAS, 380, 499

Kudoh, T., \& Basu, S. 2008, ApJ, 679, L97

Kudoh, T., \& Basu, S. 2009, Cosmic Magnetic Fields: From Planets, to Stars and Galaxies, ed. Strassmeier, K. G., et al., Cambridge:UK, 115

Langer, W. D. 1978, ApJ, 225, 95

Lee, C. W., Myers, P. C., \& Tafalla, M. 2001, ApJS, 136, 703

Li, H.-B., Dowell, C. D., Goodman, A., Hildebrand, R., \& Novak, G. 2009, ApJ, 704, 891

Li, Z.-Y., \& Nakamura, F. 2004, ApJ, 609, L83

MacLow, M.-M., \& Klessen, R. S. 2004, Rev. Mod. Phys., 76, 125

McKee, C. F., \& Ostriker, E. C. 2007, ARAA, 45, 565

Mestel, L., \& Spitzer, L. Jr. 1956, MNRAS, 116, 503

Miyama, S. M., Narita, S., \& Hayashi, C. 1987a, Prog. Theor. Phys., 78, 1051

Miyama, S. M., Narita, S., \& Hayashi, C. 1987b, Prog. Theor. Phys., 78, 1273

Mouschovias, T. Ch., \& Spitzer, L. Jr. 1976, ApJ, 210, 326

Mouschovias, T. Ch., \& Ciolek, G. E. 1999, The Origin of Stars and Planetary Systems, ed. Lada, C. J. \& Kylafis N. D., Kluwer:Dordrecht, 305

Mouschovias, T. Ch., \& Tassis, K. 2009, MNRAS, 400, 15

Nakamura, F., \& Li, Z.-Y. 2005, ApJ, 631, 411

Nakamura, F., \& Li, Z.-Y. 2008, ApJ, 687, 354

Nakano, T., \& Nakamura, T. 1978, PASJ, 30, 671

Nakano, T. 1979, PASJ, 31, 697 
Ogata, Y., Yabe, T., Shibata, K., \& Kudoh, T. 2004, International Journal of Computational Methods, 1, 201

Schleuning, D. A. 1998, ApJ, 493, 811

Shu F. H., Adams, F. C., Lizano, S. 1987, ARA\&A, 25, 23

Shu F. H., Allen, A., Shang, H., Ostriker, E. C., \& Li, Z.-Y. 1999, The Origin of Stars and Planetary Systems, ed. Lada, C. J. \& Kylafis N. D., Kluwer:Dordrecht, 193

Spitzer, L. Jr. 1942, ApJ, 95, 329

Stone, J. M., \& Norman, M. L. 1992, ApJS, 80, 791

Strittmatter, P. 1966, MNRAS, 132, 359

Tomisaka, K., Ikeuchi, S., \& Nakamura, T. 1988, ApJ, 335, 239

Yabe, T., \& Aoki, T. 1991, Comput. Phys. Commun., 66, 219

Yabe, T., \& Wang, P.-Y. 1991, J. Phys. Soc. Japan, 60, 2105

Yabe, T., Xiao, F., \& Utsumi, T. 2001, J. Comput. Phys., 169, 556

Zweibel, E. G. 1998, ApJ, 499, 746

Zweibel, E. G. 2002, ApJ, 567, 962 
Table 1. Model and Parameters

\begin{tabular}{|c|c|c|c|c|c|c|}
\hline Model & $\beta_{0}$ & $\alpha$ & Spectrum & $v_{a} / c_{s}$ & $t_{\text {core }} / t_{0}$ & Comments \\
\hline V0 & 0.25 & 0.11 & $k^{-4}$ & 0.01 & 136 & \\
\hline $\mathrm{V} 1$ & 0.25 & 0.11 & $k^{-4}$ & 0.1 & 88.9 & \\
\hline $\mathrm{V} 2$ & 0.25 & 0.11 & $k^{-4}$ & 1.0 & 41.8 & \\
\hline V3 & 0.25 & 0.11 & $k^{-4}$ & 2.0 & 24.9 & \\
\hline $\mathrm{V} 4$ & 0.25 & 0.11 & $k^{-4}$ & 3.0 & 18.7 & fiducial model \\
\hline V5 & 0.25 & 0.11 & $k^{-4}$ & 4.0 & 16.1 & \\
\hline K0 & 0.25 & 0.11 & $k^{0}$ & 0.1 & 141 & \\
\hline $\mathrm{K} 1$ & 0.25 & 0.11 & $k^{0}$ & 3.0 & 67.0 & \\
\hline $\mathrm{K} 2$ & 0.25 & 0.11 & $k^{0}$ & 5.0 & 64.9 & \\
\hline K3 & 0.25 & 0.11 & $k^{0}$ & 8.0 & 55.0 & \\
\hline $\mathrm{K} 4$ & 0.25 & 0.11 & $k^{0}$ & 10.0 & 44.7 & \\
\hline $\mathrm{A} 0$ & 0.25 & 0.0 & $k^{-4}$ & 3.0 & $>268$ & no collapse \\
\hline $\mathrm{A} 1$ & 0.25 & 0.05 & $k^{-4}$ & 3.0 & 63.6 & \\
\hline $\mathrm{A} 2$ & 0.25 & 0.2 & $k^{-4}$ & 3.0 & 3.65 & \\
\hline A3 & 0.25 & 0.05 & $k^{-4}$ & 0.1 & 220 & \\
\hline $\mathrm{A} 4$ & 0.25 & 0.2 & $k^{-4}$ & 0.1 & 44.9 & \\
\hline B0 & 0.04 & 0.11 & $k^{-4}$ & 3.0 & 29.9 & \\
\hline $\mathrm{B} 1$ & 0.09 & 0.11 & $k^{-4}$ & 3.0 & 24.8 & \\
\hline $\mathrm{B} 2$ & 0.36 & 0.11 & $k^{-4}$ & 3.0 & 6.94 & \\
\hline B3 & 4.0 & 0.11 & $k^{-4}$ & 3.0 & 1.01 & initially supercritical \\
\hline $\mathrm{B} 4$ & 9.0 & 0.11 & $k^{-4}$ & 3.0 & 0.97 & initially supercritical \\
\hline B5 & 0.04 & 0.11 & $k^{-4}$ & 0.1 & 114 & \\
\hline B6 & 0.09 & 0.11 & $k^{-4}$ & 0.1 & 108 & \\
\hline $\mathrm{B} 7$ & 0.36 & 0.11 & $k^{-4}$ & 0.1 & 68.6 & \\
\hline $\mathrm{B} 8$ & 4.0 & 0.11 & $k^{-4}$ & 0.1 & 6.93 & initially supercritical \\
\hline B9 & 9.0 & 0.11 & $k^{-4}$ & 0.1 & 5.99 & initially supercritical \\
\hline B10 & $\infty$ & 0.11 & $k^{-4}$ & 0.1 & 5.38 & initially supercritical \\
\hline
\end{tabular}

Note. - $\beta_{0}$ is the initial plasma $\beta$ at $z=0 . \alpha$ is a dimensionless ambipolar diffusion coefficient. $v_{a}$ is the amplitude of the initial velocity fluctuation. $t_{c o r e}$ is the time of collapsing core formation. 

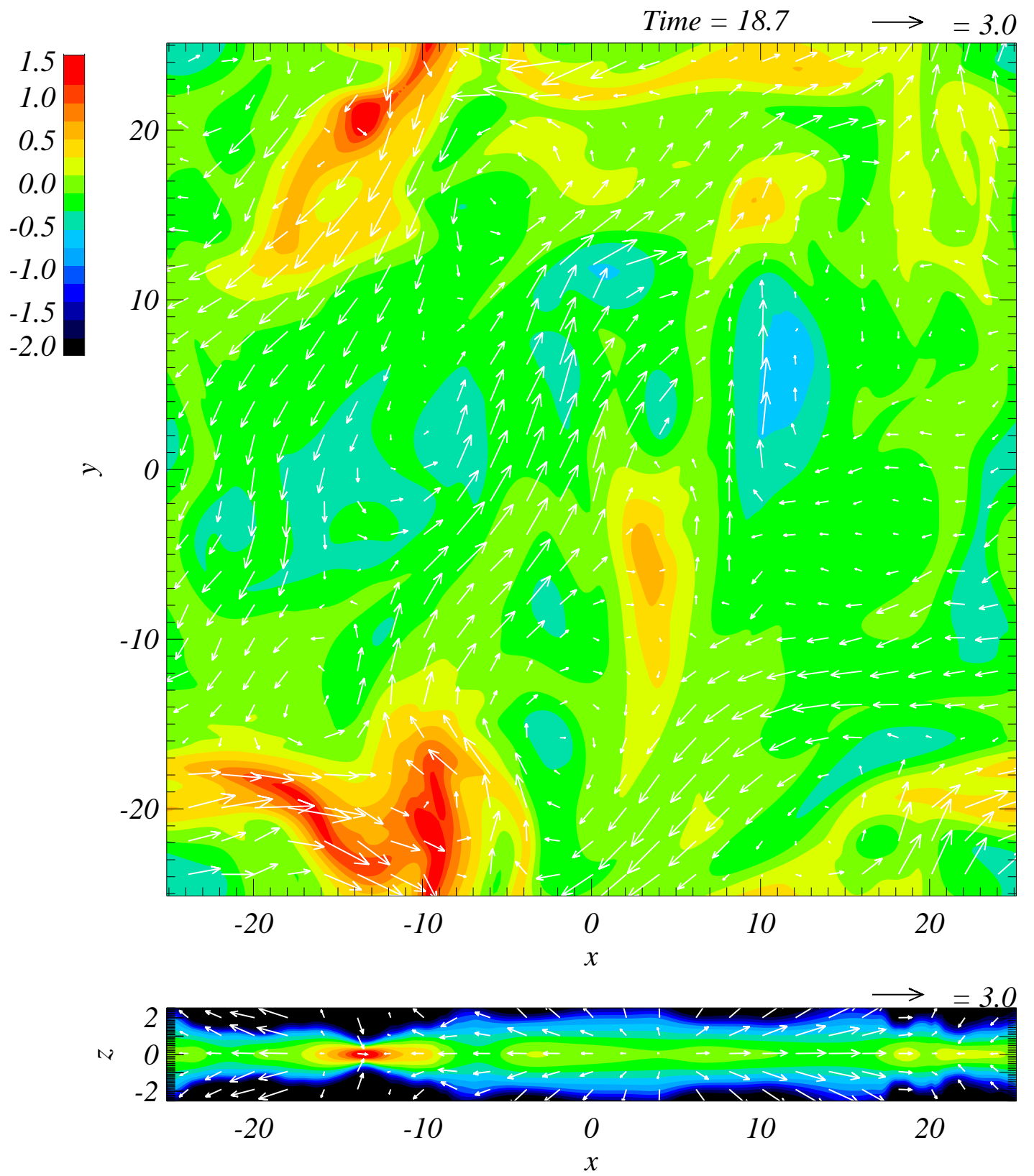

Fig. 1. - Logarithmic density contours at $t=18.7 t_{0}$ for the model V4. The model V4 is the fiducial model, in which the initial velocity amplitude $v_{a}=3 c_{s 0}$, its spectrum is $v_{k}^{2} \propto k^{-4}$, the initial normalized mass-to-flux ratio is about 0.5 (i.e., $\beta_{0}=0.25$ ), and the dimensionless ambipolar diffusion coefficient has a typical value $(\alpha=0.11)$. The unit of time $t_{0}$ is $\simeq 2.5 \times 10^{5} \mathrm{yr}$, and $H_{0}$ is $\simeq 0.05 \mathrm{pc}$. The top panel shows the $x-y$ cross section at $z=0$, and the bottom shows the $x-z$ cross section at $y=-20.7 H_{0}$. Arrows show velocity vectors on each cross section. The unit of the velocity vector is three times the sound speed $c_{s 0}$. 

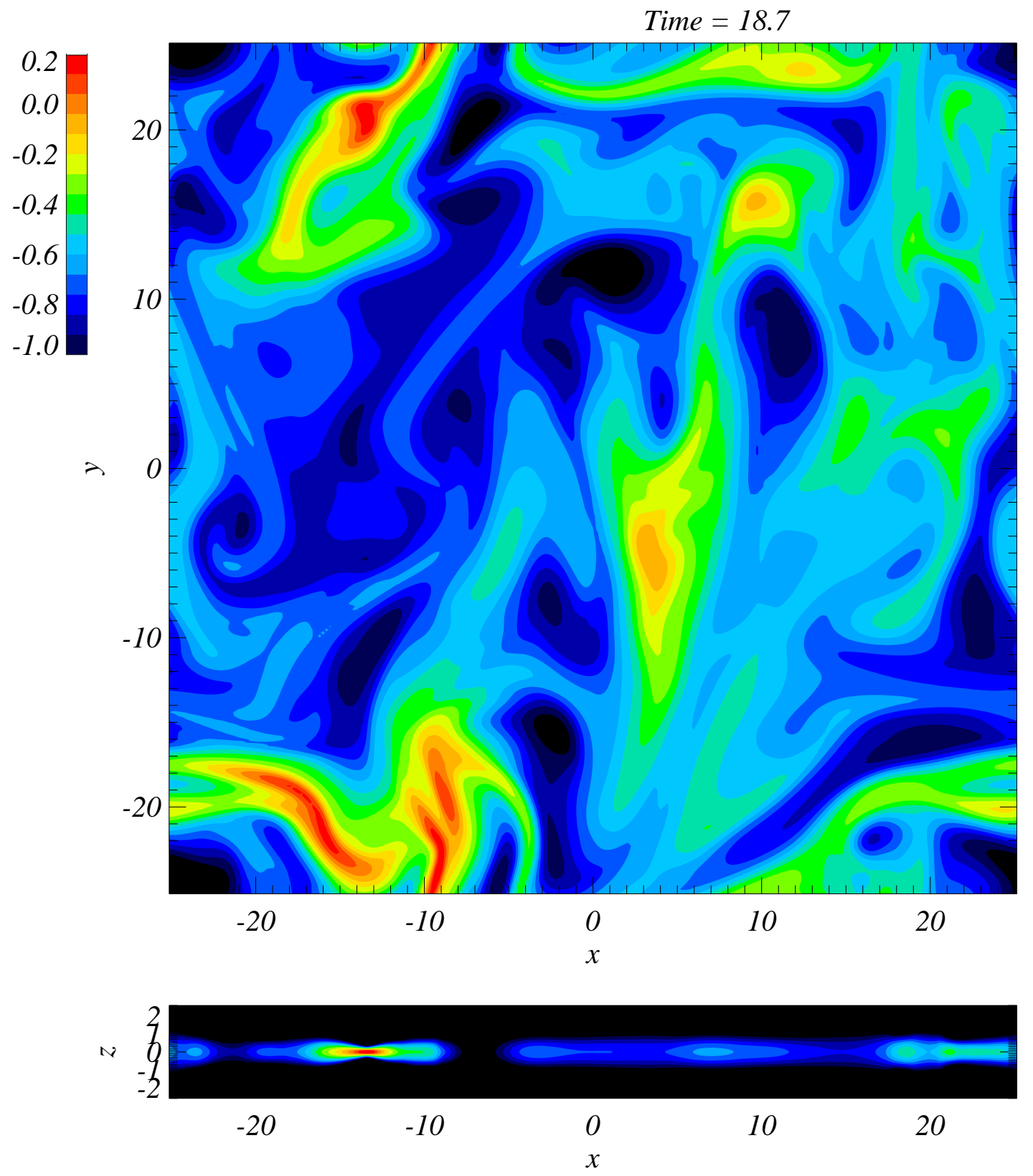

Fig. 2. - Logarithmic plasma $\beta$ contours at $t=18.7 t_{0}$ for the model V4. The top panel shows the $x-y$ cross section at $z=0$, and the bottom panel shows the $x-z$ cross section at $y=-20.7 H_{0}$. 

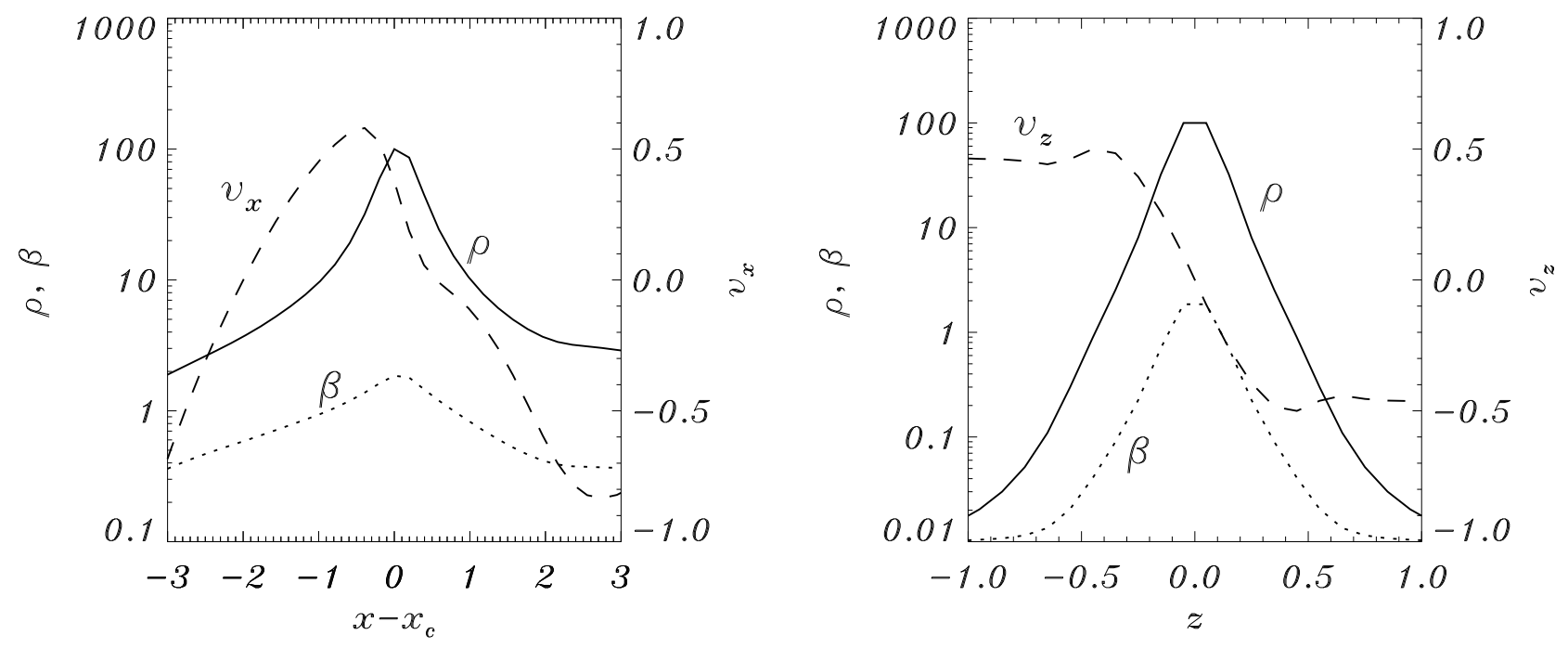

Fig. 3.- Left: The density (solid line), $x$-velocity (dashed line) and plasma $\beta$ (dotted line) along a $x$-axis cut at $y=20.7 H_{0}$ and $z=0$ in the snapshot shown in Fig. 1 and Fig. 2. The $x$-positions are measured by offset from $x_{c}=-13.4 H_{0}$, which is the maximum density point for the core. Right: The density, $z$-velocity and plasma $\beta$ along a $z$-axis cut at $x=-13.4 H_{0}$ and $y=20.7 H_{0}$ in the snapshot shown in Fig. 1 and Fig. 2. The line styles are the same as those in the left panel. The unit of length $H_{0}$ is $\simeq 0.05 \mathrm{pc}$. 


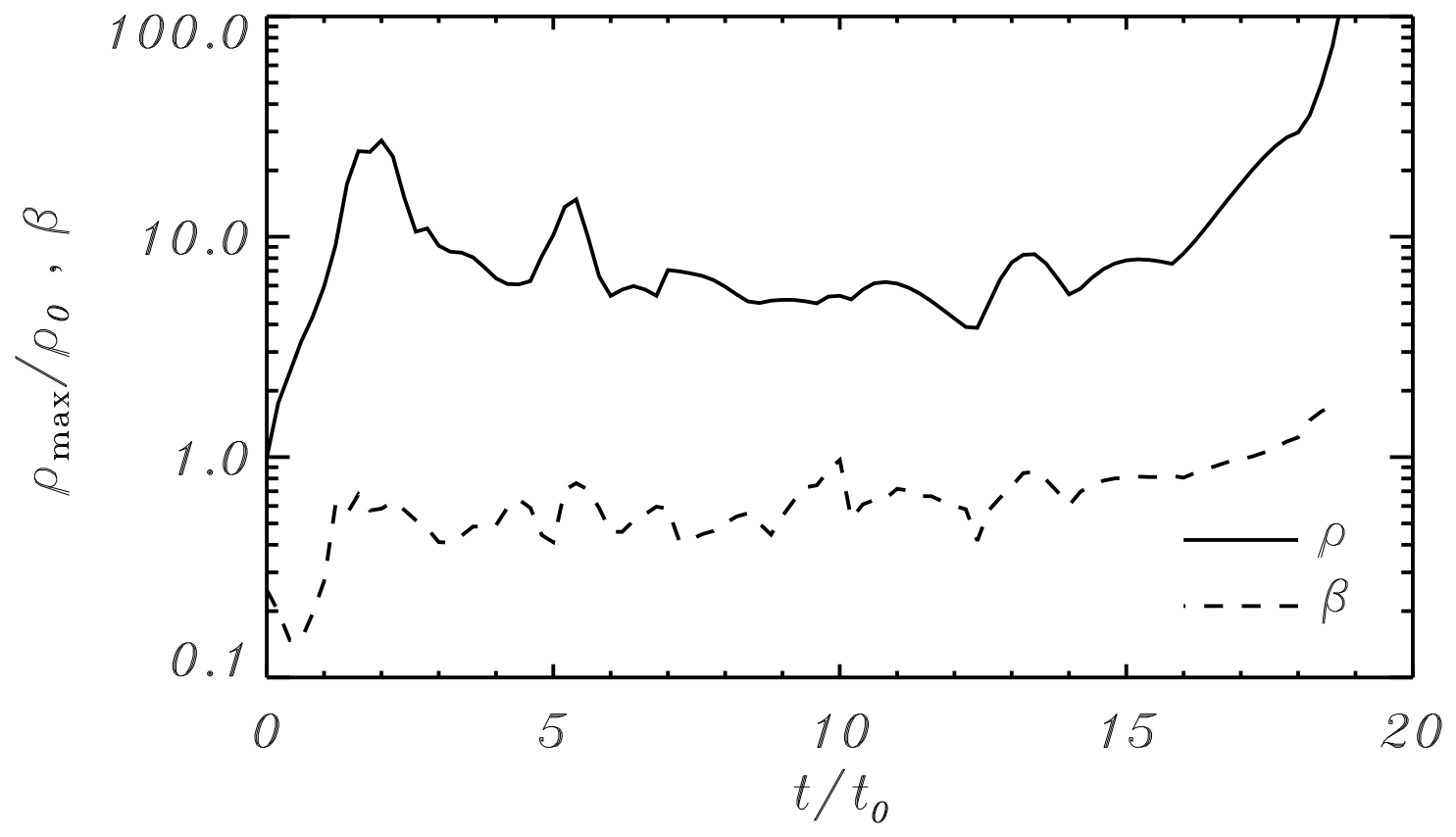

Fig. 4. - Time evolution of the maximum density (solid line) at $z=0$, and the evolution of plasma $\beta$ at the location of maximum density (dashed line) for the model V4. The unit of time $t_{0}$ is $\simeq 2.5 \times 10^{5} \mathrm{yr}$. 


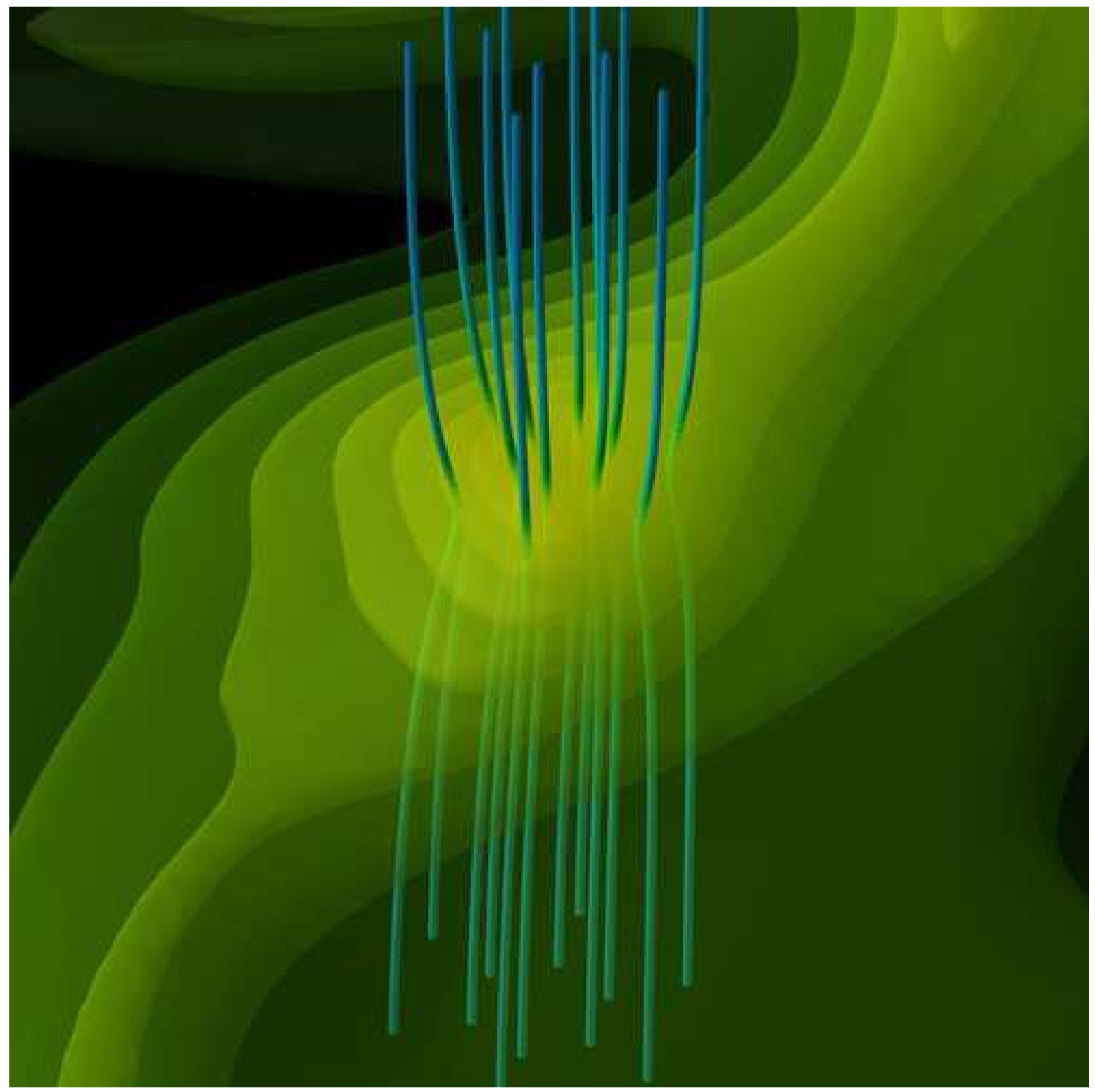

Fig. 5.- Isosurface of the logarithmic density, and the magnetic field lines near a core in the vicinity of $(x, y, z)=\left(-13.4 H_{0}, 20.7 H_{0}, 0.0\right)$ in Fig. 1. 


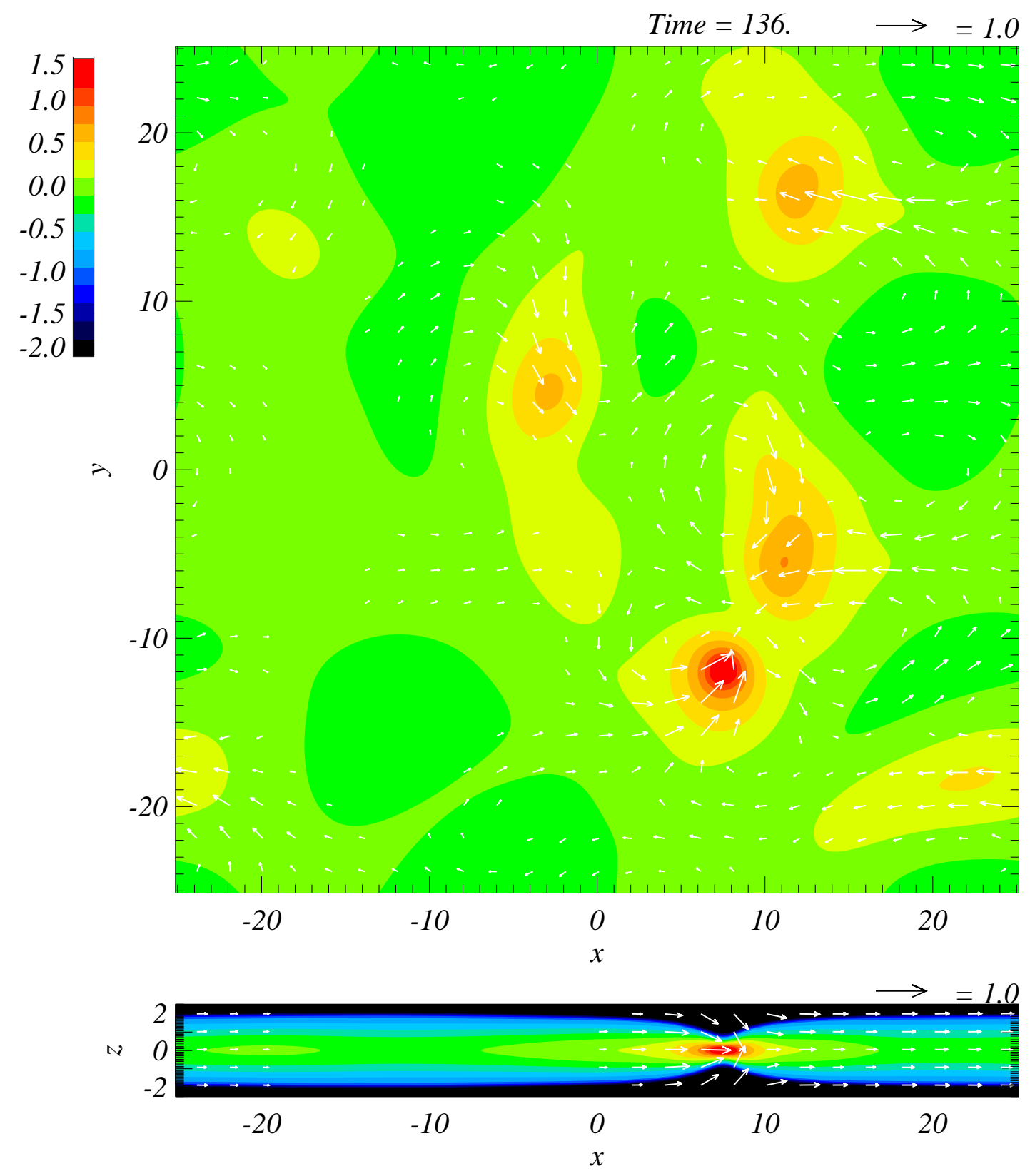

Fig. 6. - Logarithmic density contours at $t=136 t_{0}$ for the model V0. The model V0 coresponds to the model for initial velocity amplitude $v_{a}=0.01 c_{s 0}$, but other parameters are the same as the fiducial model V4. The top panel shows the $x-y$ cross section at $z=0$, and the bottom panel shows the $x-z$ cross section at $y=-11.9 H_{0}$. Arrows show velocity vectors on each cross section. The unit of the velocity vector is the sound speed $c_{s 0}$. 

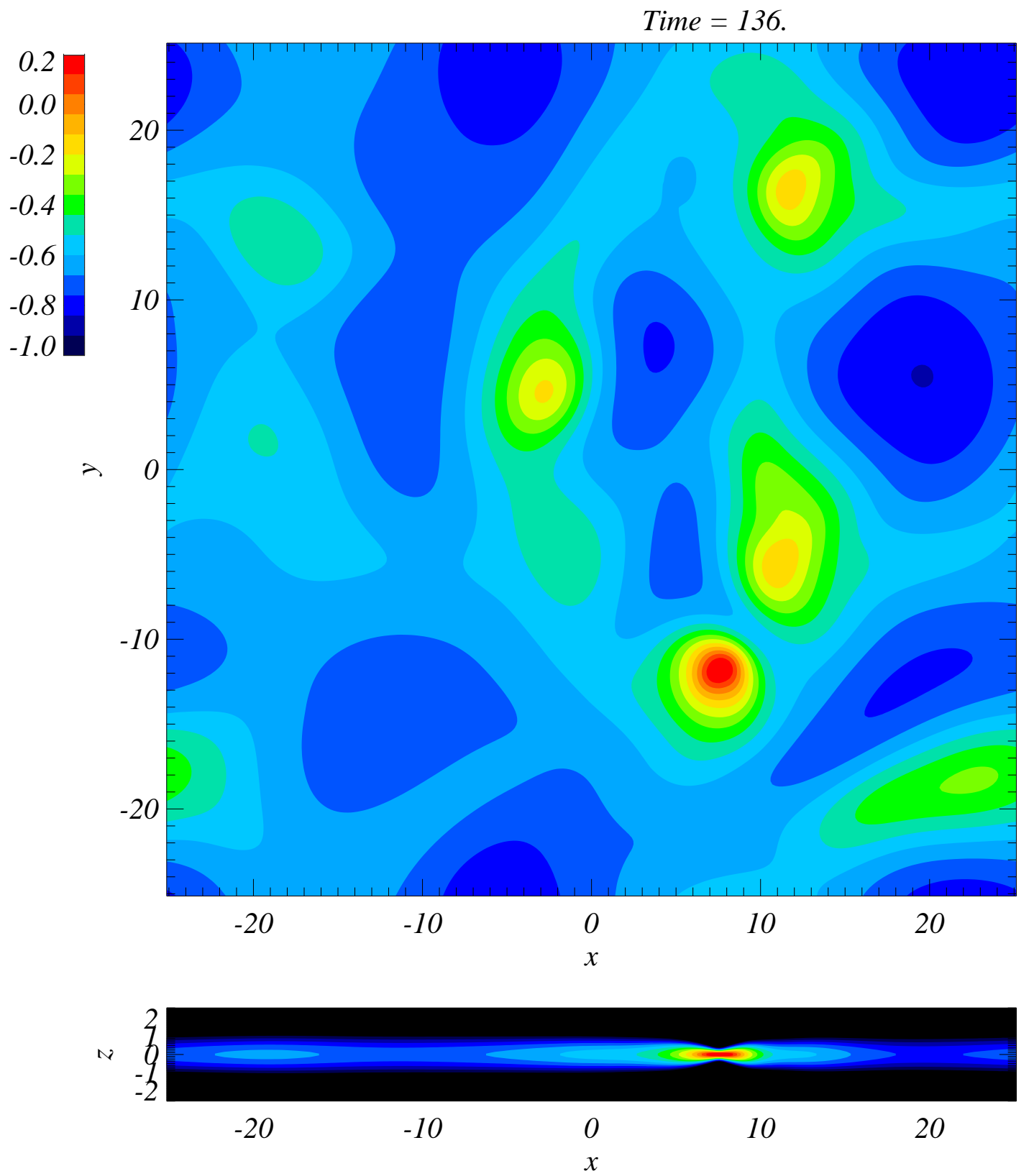

Fig. 7. - Logarithmic plasma $\beta$ contours at $t=136 t_{0}$ for the model V0. The top panel shows the $x-y$ cross section at $z=0$, and the bottom panel shows the $x-z$ cross section at $y=-11.9 H_{0}$. 

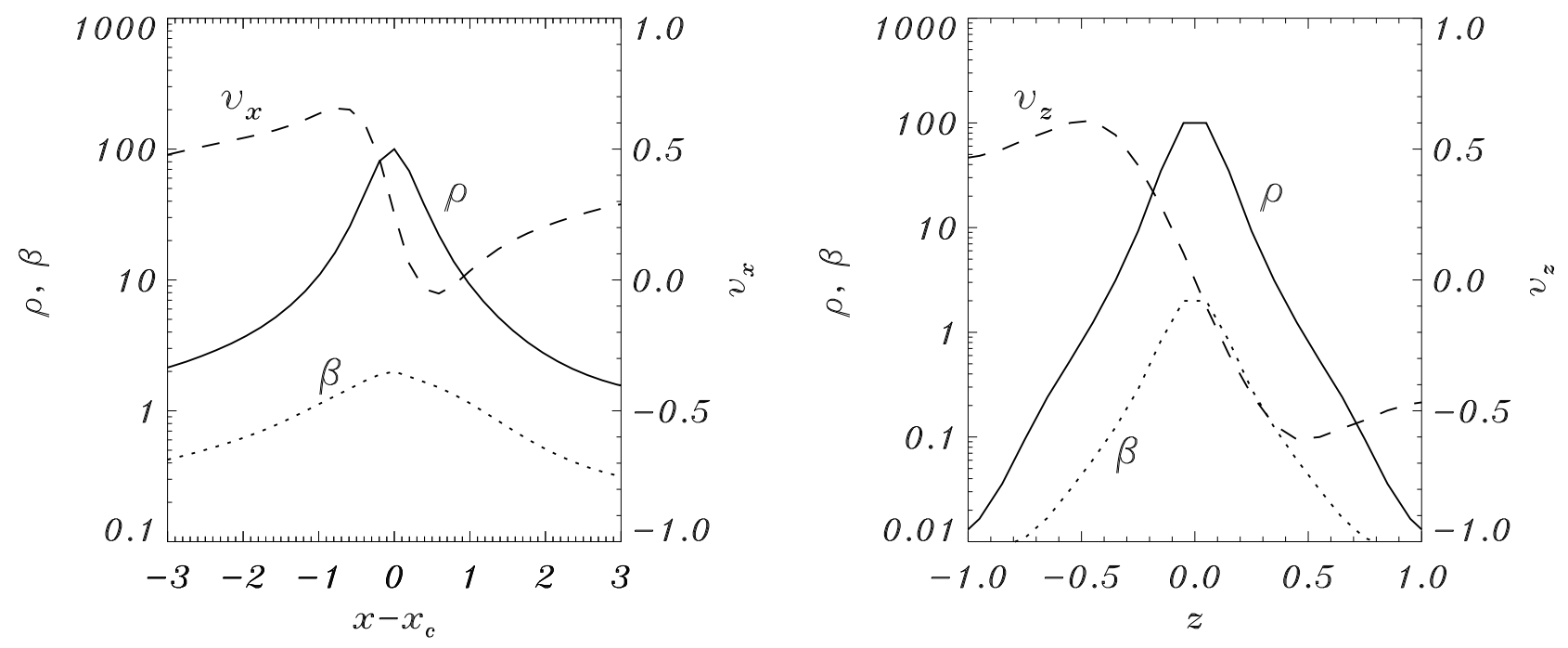

Fig. 8.- Left: The density (solid line), $x$-velocity (dashed line) and plasma $\beta$ (dotted line) along a $x$-axis cut at $y=-11.9 H_{0}$ and $z=0$ in the snapshot shown in Fig. 6 and Fig. 7. The $x$-positions are measured by offset from $x_{c}=7.6 H_{0}$, which is the maximum density point for the core. Right: The density, $z$-velocity and plasma $\beta$ along a $z$-axis cut at $x=7.6 H_{0}$ and $y=-11.9 H_{0}$ in the snapshot shown in Fig. 6 and Fig. 7. The line styles are the same as those in the left panel. The unit of length $H_{0}$ is $\simeq 0.05 \mathrm{pc}$. 


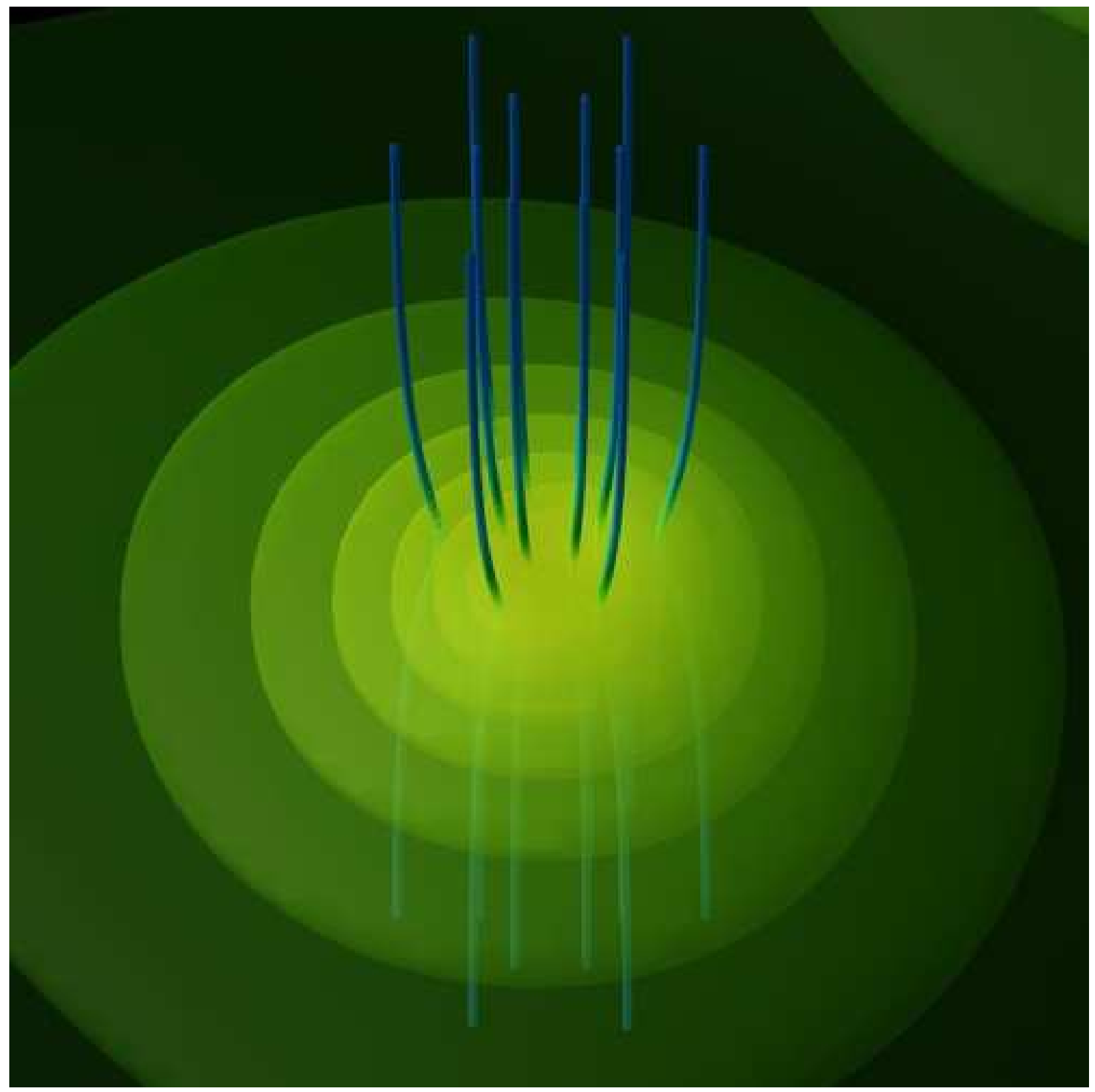

Fig. 9.- Isosurface of the logarithmic density and magnetic field lines near the core in the vicinity of $(x, y, z)=\left(7.6 H_{0},-11.9 H_{0}, 0.0\right)$ in Fig. 6 . 


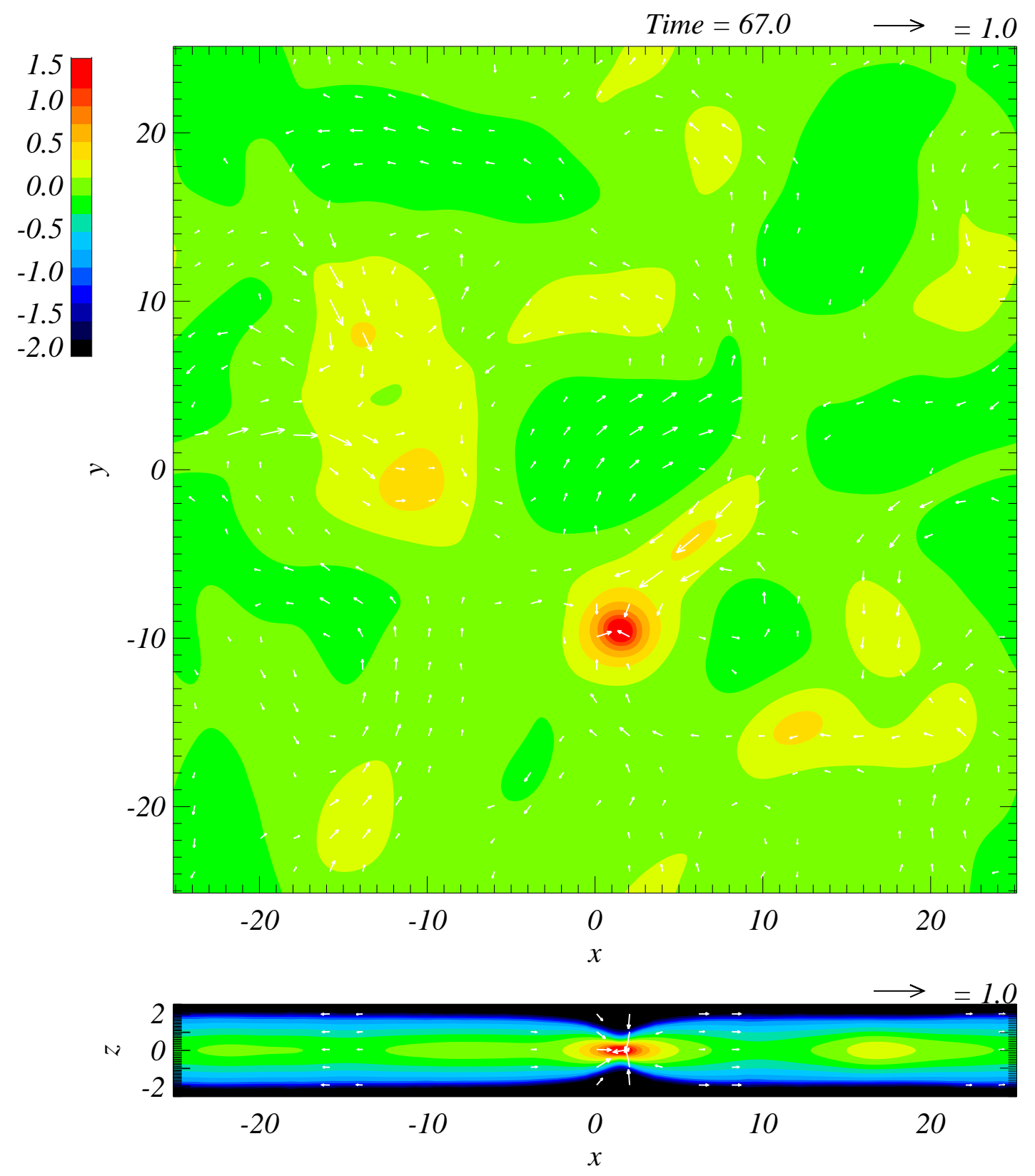

Fig. 10. - Logarithmic density contours at $t=67.0 t_{0}$ for the model K1. The model K1 has an initial velocity spectrum $v_{k}^{2} \propto k^{0}$, but other parameters are the same as in the fiducial model V4. The top panel shows the $x-y$ cross section at $z=0$, and the bottom panel shows the $x-z$ cross section at $y=-9.5 H_{0}$. Arrows show velocity vectors on each cross section. The unit of the velocity vector is the sound speed $c_{s 0}$. 

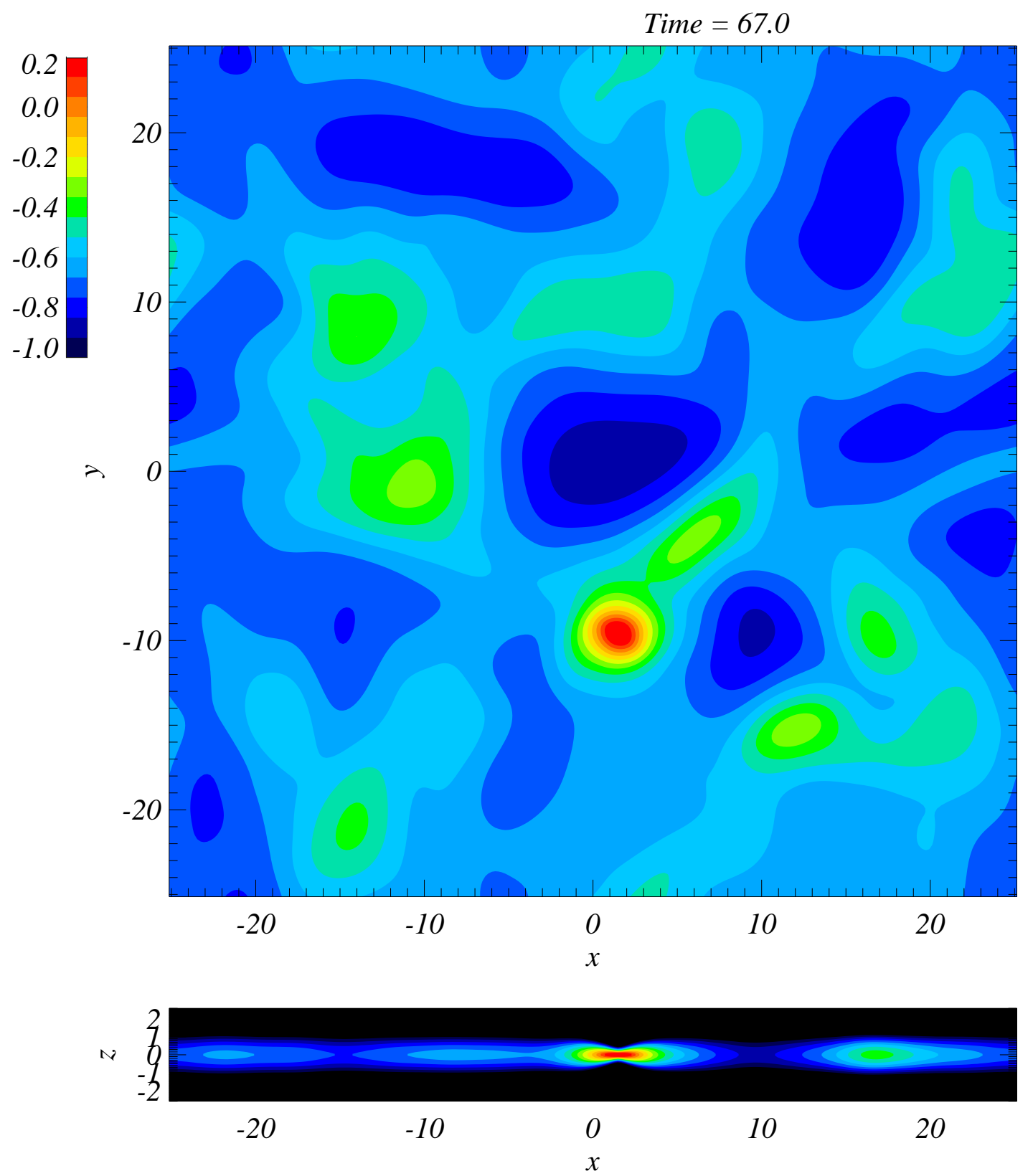

Fig. 11. - Logarithmic plasma $\beta$ contours at $t=67.0 t_{0}$ for the model K1. The top panel shows the $x-y$ cross section at $z=0$, and the bottom panel shows the $x-z$ cross section at $y=-9.5 H_{0}$. 

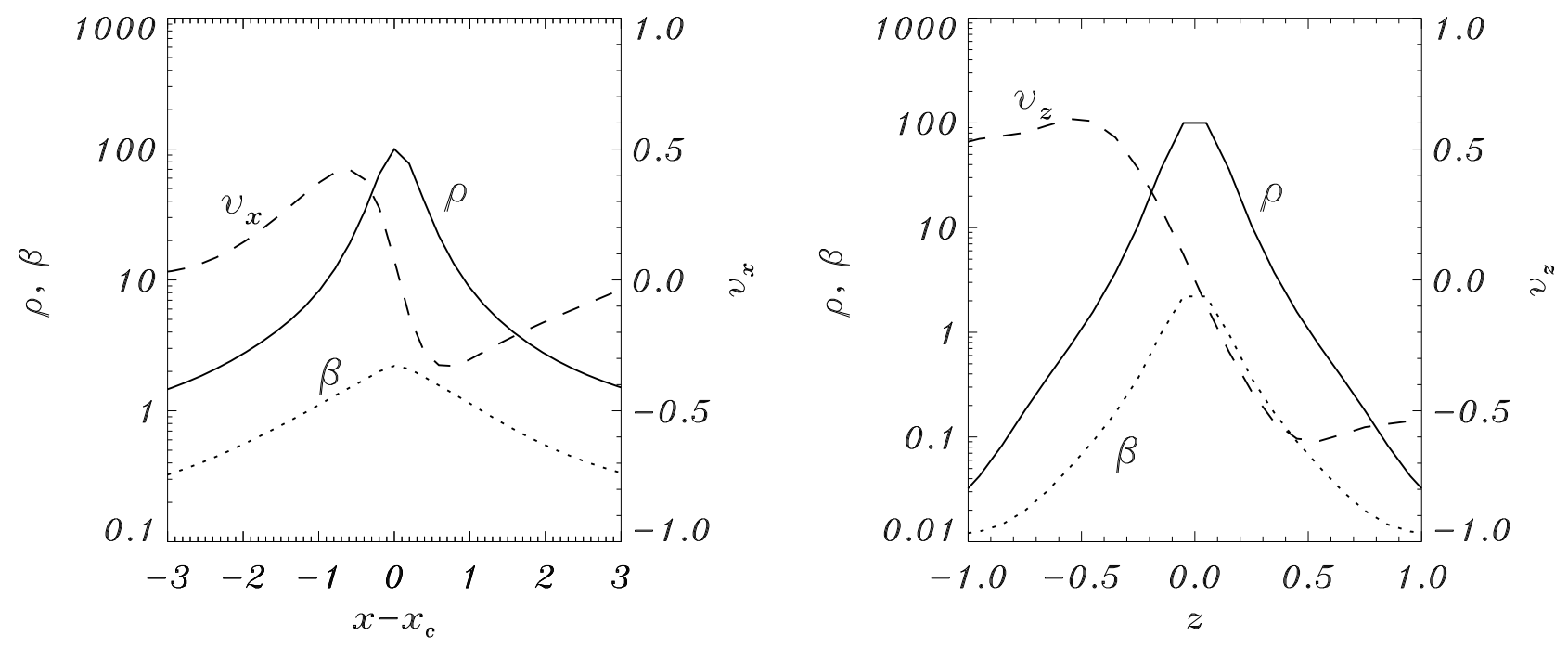

Fig. 12.- Left: The density (solid line), $x$-velocity (dashed line) and plasma $\beta$ (dotted line) along a $x$-axis cut at $y=-9.5 H_{0}$ and $z=0$ in the snapshot shown in Fig. 10 and Fig. 11. The $x$-positions are measured by offset from $x_{c}=-1.5 H_{0}$, which is the maximum density point for the core. Right: The density, $z$-velocity and plasma $\beta$ along a $z$-axis cut at $x=-1.5 H_{0}$ and $y=-9.5 H_{0}$ in the snapshot shown in Fig. 10 and Fig. 11. The line styles are the same as those in the left panel. The unit of length $H_{0}$ is $\simeq 0.05 \mathrm{pc}$. 


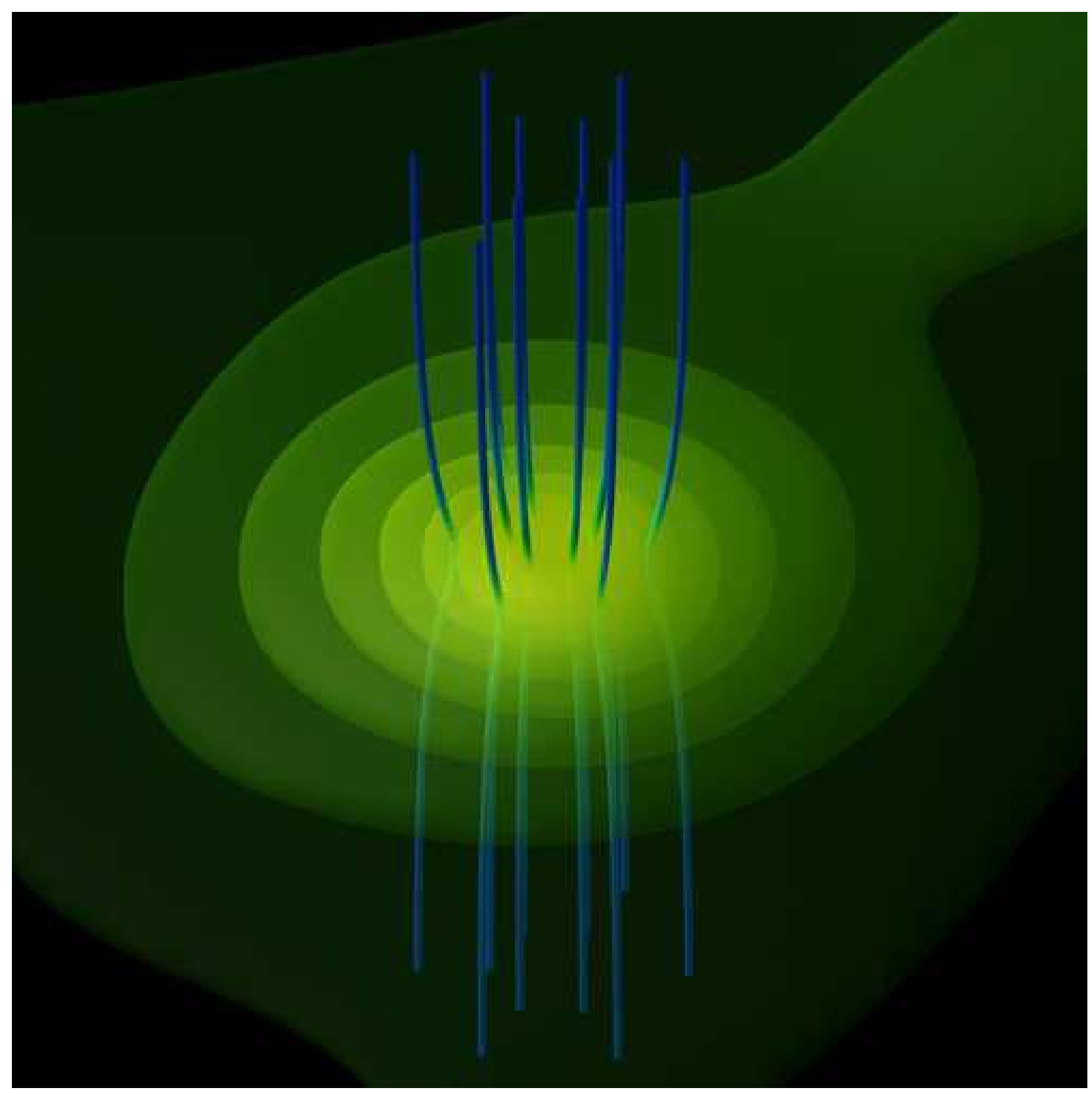

Fig. 13.- Isosurface of the logarithmic density and magnetic field lines near the core in the vicinity of $(x, y, z)=\left(-1.5 H_{0},-9.5 H_{0}, 0.0\right)$ in Fig. 10. 


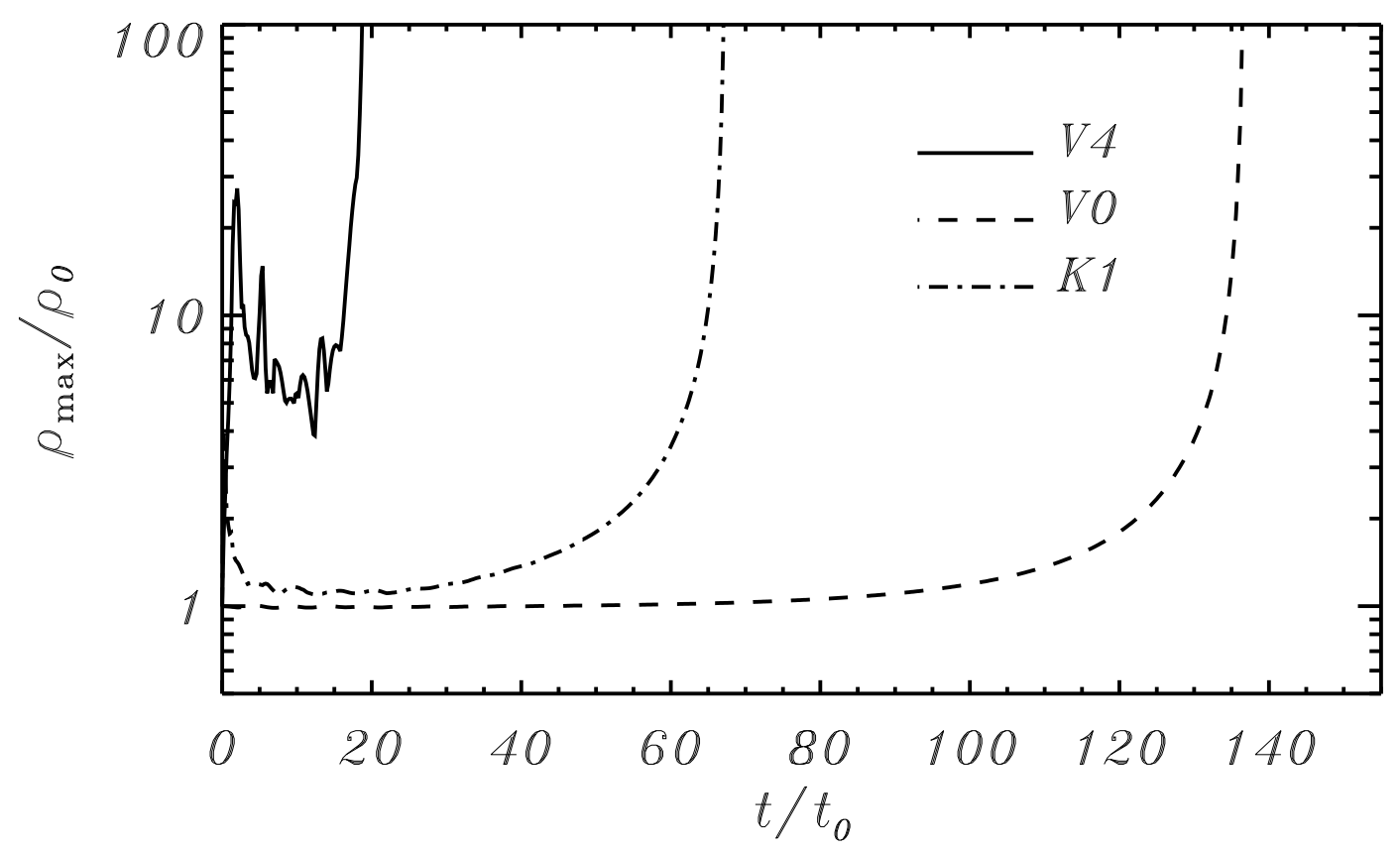

Fig. 14. - Time evolution of the maximum density at $z=0$ for the model V4 (solid line), V0 (dashed line), and K1 (dash-dotted line), showing the difference between models with different velocity perturbation. The model V4 is the fiducial model, in which $v_{a}=3 c_{s 0}$, its spectrum is $v_{k}^{2} \propto k^{-4}$, the initial normalized mass-to-flux ratio is about 0.5 (i.e., $\beta_{0}=0.25$ ), and the dimensionless ambipolar diffusion coefficient has a typical value $(\alpha=0.11)$. The model V0 has $v_{a}=0.01 c_{s 0}$, but other parameters are the same as those of model V4. The model K1 has a velocity spectrum $v_{k}^{2} \propto k^{0}$, but other parameters are the same as those of model V4. The unit of time $t_{0}$ is $\simeq 2.5 \times 10^{5} \mathrm{yr}$. 


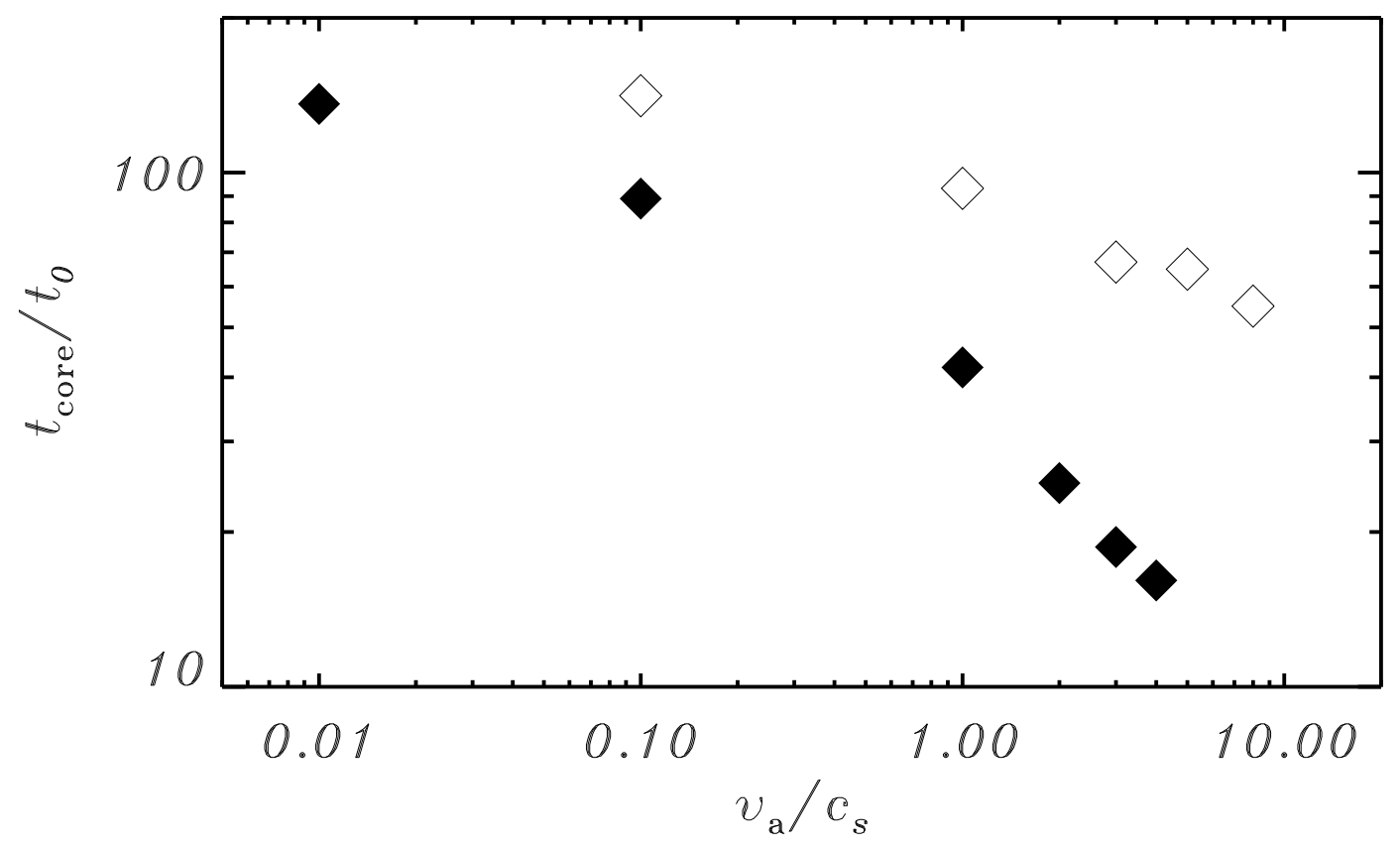

Fig. 15. - Core formation time as a function of the initial velocity amplitude. The filled squares represent the results for the initial $k^{-4}$ spectrum (model V0, V1, V2, V3, V4 and $\mathrm{V} 5$ ), and the open squares represent those for the initial $k^{0}$ spectrum (model K0, K1, K2, $\mathrm{K} 3$ and K4). The unit of time $t_{0}$ is $\simeq 2.5 \times 10^{5} \mathrm{yr}$. 


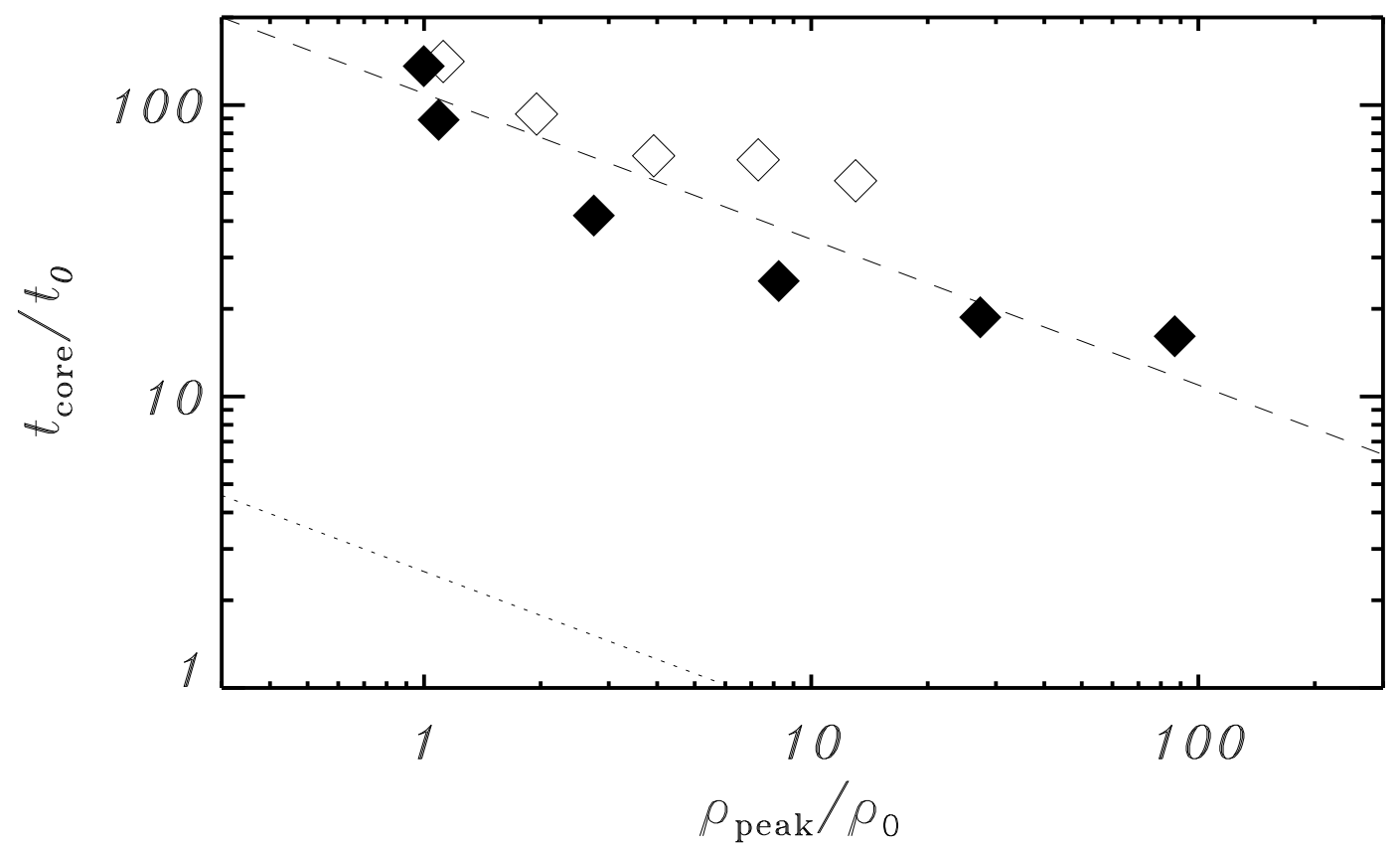

Fig. 16.- Core formation time as a function of the density peak during the first compression in its time evolution. The filled squares represent the results for the initial $k^{-4}$ spectrum (model V0, V1, V2, V3, V4 and V5), and the open squares represent those for the initial $k^{0}$ spectrum (model K0, K1, K2, K3 and K4). The dashed line shows that the core formation time is nearly proportional to $1 / \sqrt{\rho_{\text {peak }}}$. The dotted line represents the free fall time of gas with density $\rho_{\text {peak }}\left(=1 / \sqrt{G \rho_{\text {peak }}}\right)$ for comparison. The unit of time $t_{0}$ is $\simeq 2.5 \times 10^{5} \mathrm{yr}$. 


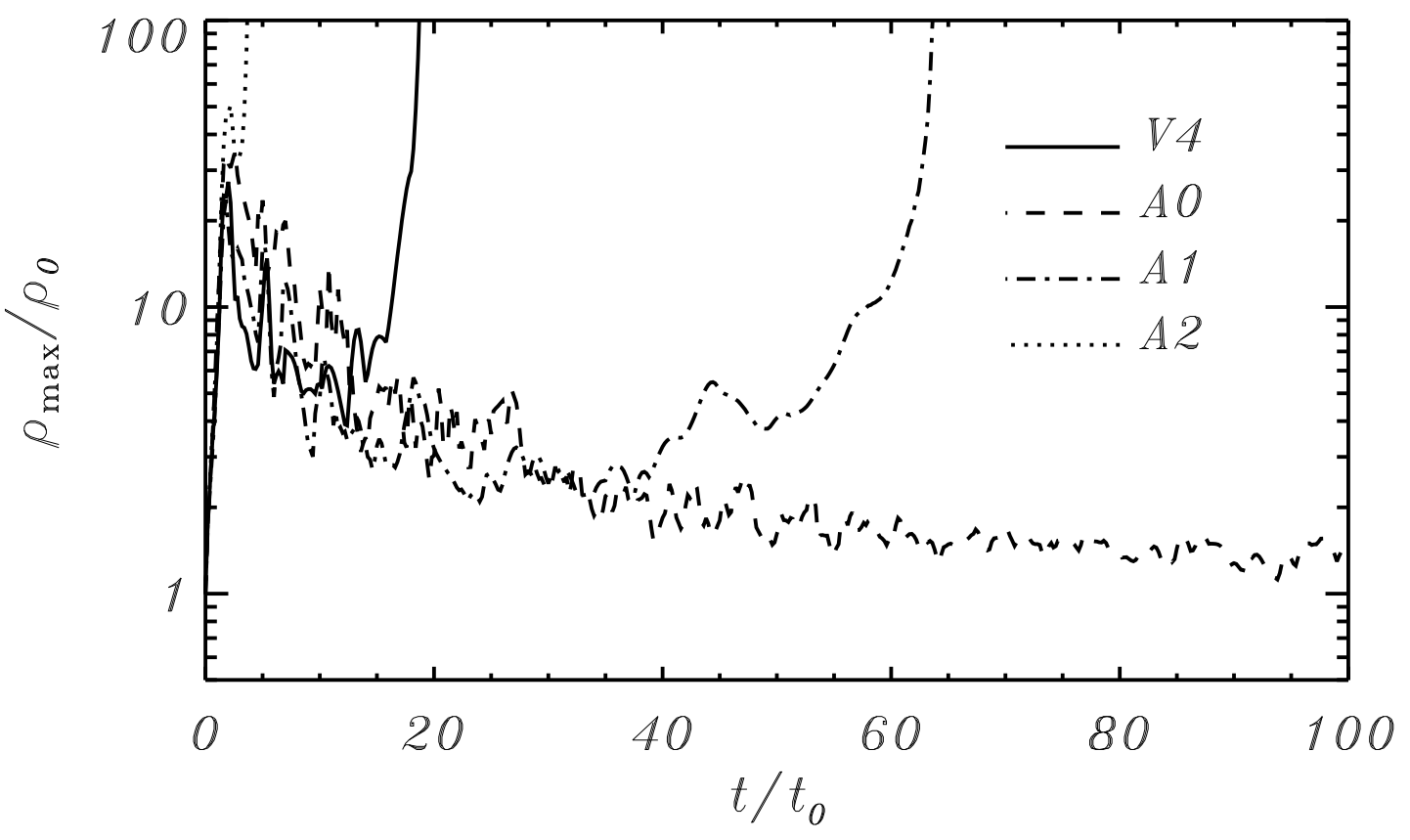

Fig. 17. - Time evolution of the maximum density at $z=0$ for the model V4 (solid line), A0 (dashed line), A1 (dash-dotted line) and A2 (dotted line), showing the difference between models with different $\alpha$. The model V4 is the fiducial model, in which the dimensionless ambipolar diffusion coefficient has a typical value $(\alpha=0.11)$. The model A0, A1, and A2 correspond to the models for $\alpha=0, \alpha=0.05$, and $\alpha=0.2$, respectively, but other parameters are the same as those of model V4. The unit of time $t_{0}$ is $\simeq 2.5 \times 10^{5} \mathrm{yr}$. 


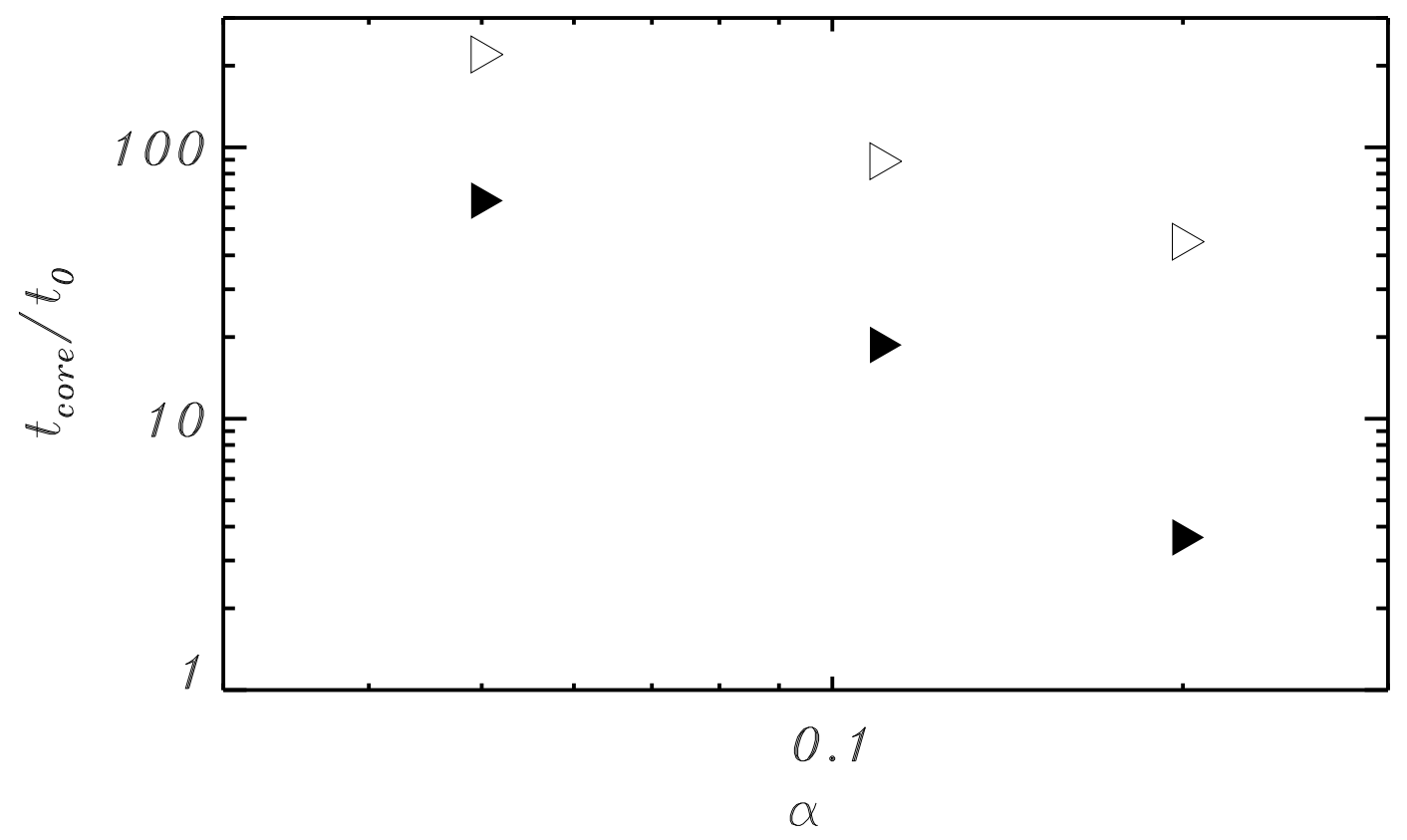

Fig. 18. - Core formation time as a function of $\alpha$, the dimensionless ambipolar diffusion coefficient. The filled triangles represent the results for an initial velocity fluctuation $v_{a}=$ $3.0 c_{s}$ (model A1, A2 and V4), and the open triangles represent those for an initial velocity fluctuation $v_{a}=0.1 c_{s}$ (model A3, A4, and V1). The unit of time $t_{0}$ is $\simeq 2.5 \times 10^{5} \mathrm{yr}$. 


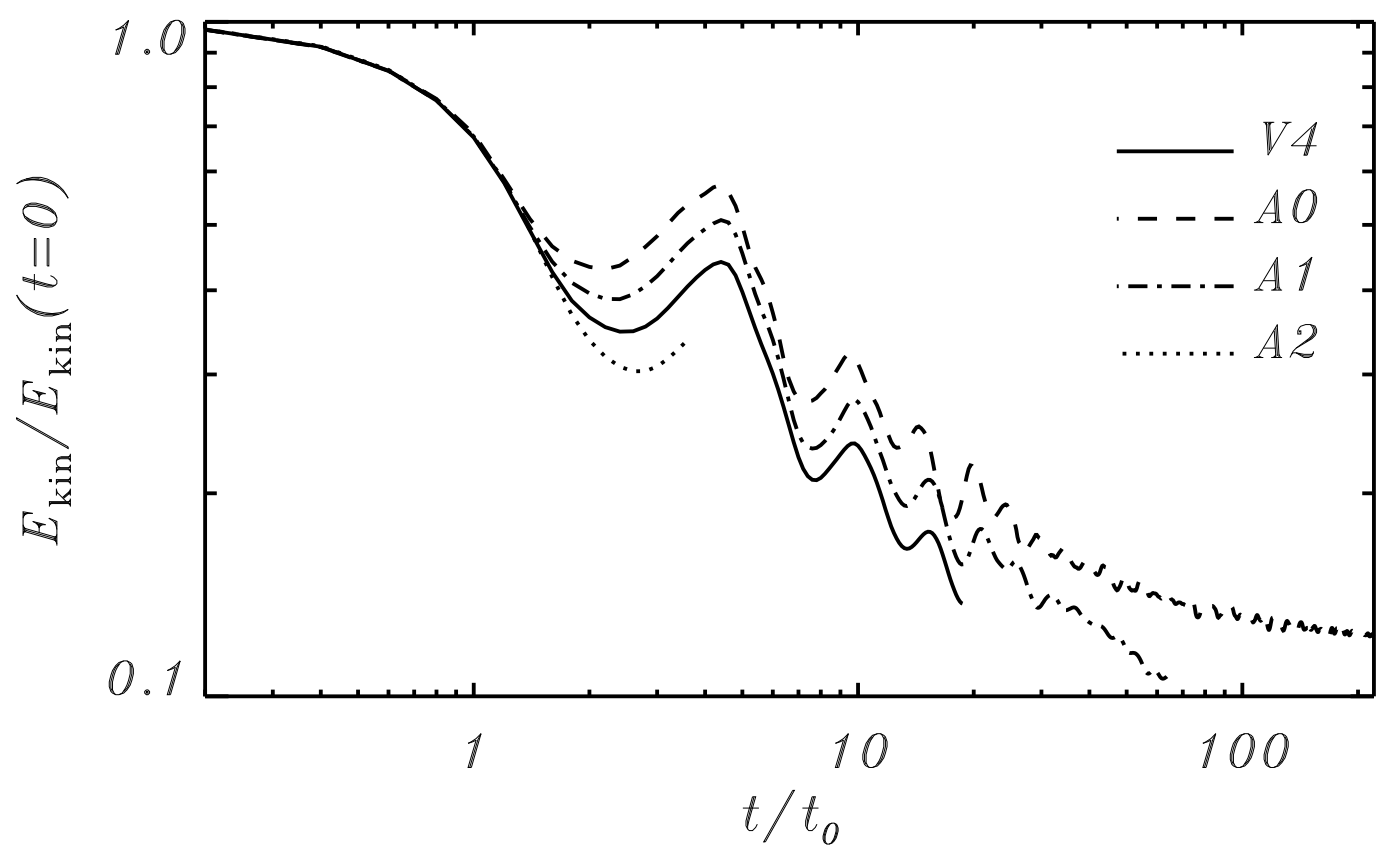

Fig. 19. - Time evolution of the total kinetic energies for the models V4 (solid line), A0 (dashed line), A1 (dash-dotted line) and A2 (dotted line), showing the difference between models with different $\alpha$. The model V4 is the fiducial model, in which the dimensionless ambipolar diffusion coefficient has a typical value $(\alpha=0.11)$. The models $\mathrm{A} 0, \mathrm{~A} 1$, and A2 correspond to $\alpha=0, \alpha=0.05$, and $\alpha=0.2$, respectively, but other parameters are the same as those of model V4. The unit of time $t_{0}$ is $\simeq 2.5 \times 10^{5} \mathrm{yr}$. 


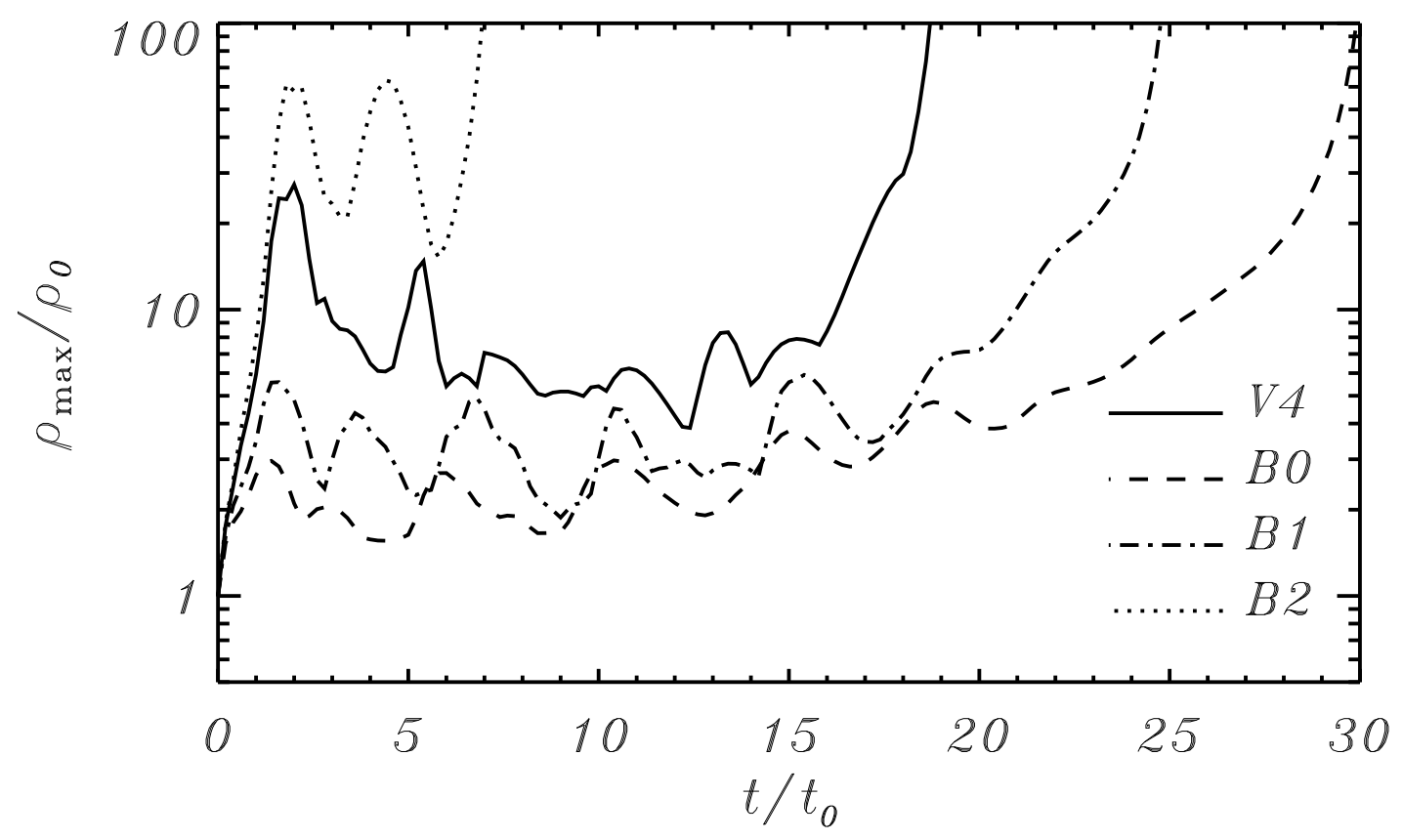

Fig. 20.- Time evolution of the maximum density on $z=0$ for the models V4 (solid line), B0 (dashed line), B1 (dash-dotted line) and B2 (dotted line), showing the difference between models with different $\beta_{0}$. The model V4 is the fiducial model, in which the initial normalized mass-to-flux ratio is about 0.5 (i.e., $\beta_{0}=0.25$ ). The models $\mathrm{B} 0, \mathrm{~B} 1$, and $\mathrm{B} 2$ correspond to $\beta=0.04, \beta=0.09$, and $\beta=0.36$, respectively, but other parameters are the same as those of model V4. The unit of time $t_{0}$ is $\simeq 2.5 \times 10^{5} \mathrm{yr}$. 


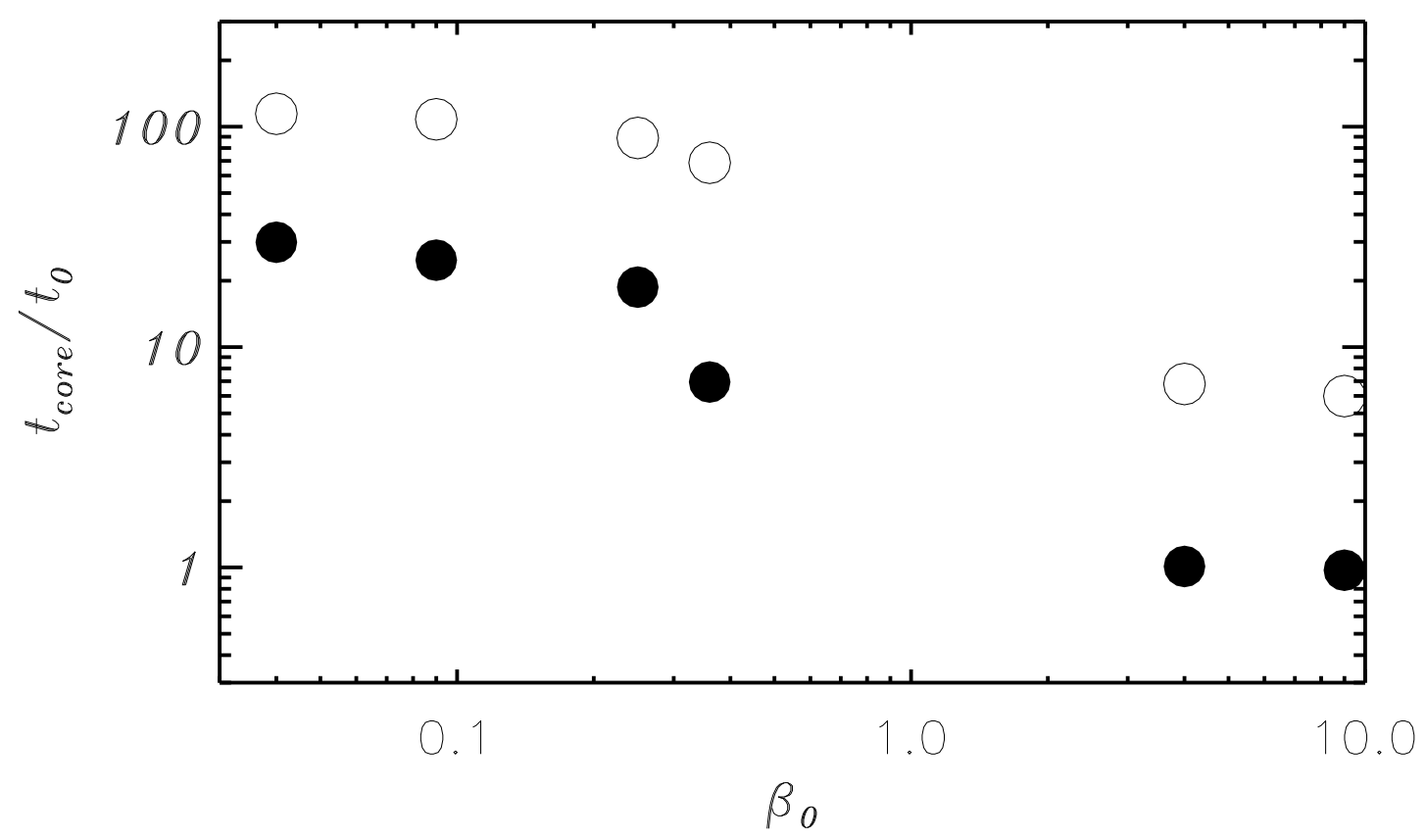

Fig. 21. - Core formation time as a function of $\beta_{0}$, the initial plasma $\beta$, at $z=0$. The filled circles represent the results for an initial velocity fluctuation $v_{a}=3.0 c_{s}$ (models B0, B1, B2, B3, B4 and V4), and the open circles represent those for an initial velocity fluctuation $v_{a}=0.1 c_{s}$ (models B5, B6, B7, B8, B9 and V1). The unit of time $t_{0}$ is $\simeq 2.5 \times 10^{5} \mathrm{yr}$. 